\title{
Identifying Microstructural Changes in Diffusion MRI; How to Break
}

\section{Parameter Degeneracy}

\author{
Hossein Rafipoor ${ }^{1}$, Ying-Qiu Zheng ${ }^{1}$, Ludovica Griffanti ${ }^{1,2}$, Saad Jbabdi ${ }^{1}$, and Michiel Cottaar ${ }^{1}$ \\ ${ }^{1}$ Wellcome Centre for Integrative Neuroimaging, FMRIB, Nuffield Department of Clinical \\ Neurosciences, Oxford, UK \\ ${ }^{2}$ Wellcome Centre for Integrative Neuroimaging, Oxford Centre for Human Brain Activity, \\ Department of Psychiatry, University of Oxford, Oxford, UK
}

\begin{abstract}
Biophysical models that attempt to infer real-world quantities from data usually have many free parameters. This over-parameterisation can result in degeneracies in model inversion and render parameter estimation ill-posed. However, often, we are not interested in estimating the parameters per se, but rather in identifying changes in parameters between experimental conditions (e.g. patients vs controls). Here we present a Bayesian framework to make inference on changes in the parameters of biophysical models even when model inversion is degenerate, which we refer to as Bayesian EstimatioN of CHange (BENCH).

We infer the parameter changes in two steps; First, we train models that can estimate the pattern of change in the measurements given any hypothetical change in the parameters using simulations. Next, for any pair of real data sets, we use these pre-trained models to estimate the probability that an observed difference in the data can be explained by each model of change.

The approach is general and particularly useful for biophysical models with parameter degeneracies. In this paper, we apply the approach in the context of microstructural modelling of diffusion MRI data, where the models are usually over-parameterised and not invertible without injecting strong assumptions. Using simulations, we show that in the context of the standard
\end{abstract}


model for diffusion our approach is able to identify changes in microstructural parameters from multi-shell diffusion MRI data. We also apply our approach to a subset of subjects from the UK-Biobank Imaging to identify the dominant standard model parameter change in areas of white matter hyperintensities.

\section{INTRODUCTION}

Modelling diffusion MRI (dMRI) data comes in two flavours. Phenomenological models, such as diffusion tensor imaging (DTI) (Basser et al. 1994) and DKI (Jensen et al. 2005)) attempt to capture the structure of the data, while (bio)physical models such as the standard model (Novikov et al. 2019a), NODDI (Zhang et al. 2012), Ball and Rackets (Sotiropoulos et al. 2012) and AxCaliber (Assaf et al.2008)) attempt to infer properties of the tissue microstructure given the data. This active field of research relies on the inversion of biophysical forward models, but it is also notoriously difficult to overcome model degeneracies (Jelescu et al. 2016). To resolve these degeneracies, the conventional approach is to constrain a subset of the parameters and only make inferences on the remaining parameters (Zhang et al. 2012). However, the validity of the extra assumptions, specifically under different experimental conditions is unclear. As a result, not only is there a limit to the number of microstructural parameters that can be estimated, but the reliability of the estimated parameters can also be questionable (Jelescu et al. 2016; Reisert et al. 2017; Lampinen et al. 2019).

However, in many real-world applications, the model parameters may not be of direct interest. Rather, we are often interested in the "change" in the parameters under different experimental conditions. For example, to study mechanisms underlying a disease one would normally compare the parameter estimates of biophysical models between patient and control groups, with little attention paid to the actual parameter estimates. However, the parameter estimation is only tractable when the model of interest is invertible given the data. This limits one to simple biophysical models or requires injection of prior assumptions.

In this work, we show that we can make precise inferences on the change in model parameters even in complex degenerate models. We argue that, using a sparsity assumption on the pattern 

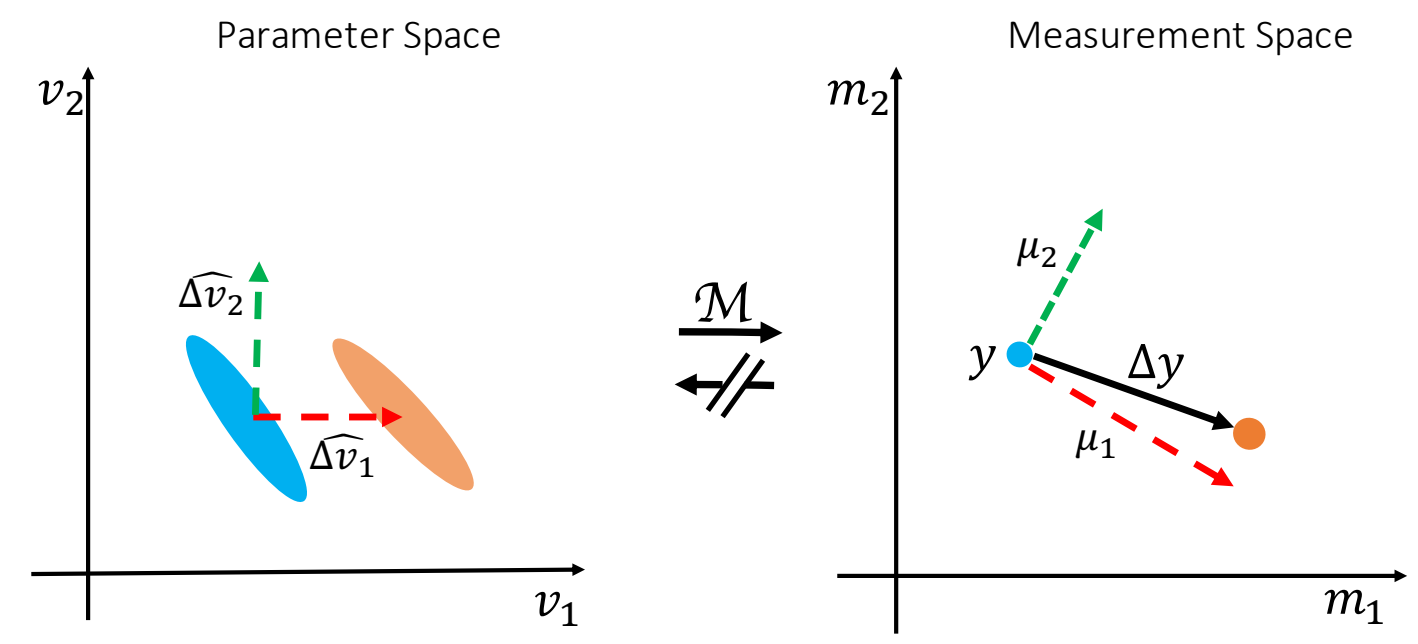

Figure 1. Illustration of the inversion-free inference on change (BENCH). Consider a toy model with two parameters and two measurements $\mathcal{M}\left(v_{1}, v_{2}\right)=\left[m_{1}, m_{2}\right]$. Each oval in the parameter space (left) corresponds to a single point in the measurement space (right) with the same color; meaning that there is a one to many mapping from measurements to parameters (i.e., the model is degenerate). Despite the degeneracies we are able to estimate which of the parameters best explains the change in the measurements. We do so by comparing the observed change $(\Delta y)$ with the expected change in the measurements $\left(\mu_{1}, \mu_{2}\right)$ as a result of each hypothesised pattern of change $\left(\Delta \hat{v}_{1}, \Delta \hat{v}_{2}\right)$.

of change, we can limit the hypothesis space, and so circumvent the degeneracy in the parameter estimation (see Figure 1). Our approach proceeds in two steps: First, we use simulated data generated from a forward model to train models that calculate how each parameter affects the measurements. Once these models of change have been trained for all hypothetical patterns of change, we use them to infer the posterior probability of which parameter(s) can best explain the change between real datasets. We call this approach $\mathrm{BENCH}$, which stands for Bayesian EstimatioN of CHange.

When confronted with a degenerate biophysical model, BENCH makes a different set of assumptions from the traditional approach of fixing some parameters and identifying any change in the remaining free parameters. When comparing patients and controls, the traditional approach assumes that the prior values for the fixed parameters hold across the region of interest in both groups. Hence, any change of signal across the region of interest between the two groups is assumed to be fully explained by the predetermined set of free parameters. In contrast, by not relying on model inversion, BENCH can work directly with the degenerate biophysical model without fixing any parameters. However, this comes at the price of limiting the patterns of change to some 
predetermined set of possible patterns of change set by the user (e.g., parameter A could change, or parameter B increases by the same amount as parameter $\mathrm{C}$ decreases). While the number of such proposed microstructural changes can be large, each of them has to be sparse (i.e., they have a lower degree of freedom than the number of free parameters that could be estimated in the traditional approach). In this work, we will limit ourselves to changes of just one parameter at a time.

$\mathrm{BENCH}$ is a general framework that is applicable to any situation where we are interested in comparing parameters of a generative (bio)physical model across different conditions. Here we apply the framework to dMRI microstructure modelling. As an example use case, we studied microstructural changes in White Matter Hyperintensities (WMH), which are extra bright regions that are commonly seen in T2-weighted images at specific brain regions in elderly people. Despite the abundance and clinical implications of WMHs (Prins and Scheltens 2015; Debette and Markus 2010), the underlying changes in the histopathology and microstructure remain unknown (Wardlaw et al. 2013).

The structure of this paper is as follows. In the Theory section, we present the general inference method and how we train the models of change. In the Methods section, we cover the diffusionspecific materials including the computation of summary measurements that are used to represent diffusion data and the microstructural model for diffusion MRI. In the Results section, we first demonstrate the ability of our model in detecting the underlying parameter changes using simulated data. We then apply the method to study microstructural changes in white matter hyperintensities as an example application. In the Discussion section, the potential applications, limitations, and possible future directions of this work are presented.

\section{THEORY}

\section{Inference on change in parameters}

Given a baseline measurement (y), an observed change in the measurement $(\Delta y)$, and a generative biophysical model $(\mathcal{M})$, we aim to investigate what pattern of change $(\hat{\Delta v})$ in the model parameters $(v)$ can best explain this observed change in the measurements (Figure 1). A pattern of change is a unit vector in the parameter space, e.g. it can be a change in a single parameter, or 
any linear combination of the model parameters. For simplicity of the explanations and notation, we only assume a single parameter change in the rest of paper, but all the equations apply to any linear combination of the parameters. If the model is invertible, we may directly estimate $\Delta v$ by inverting the model on $y$ and $y+\Delta y$ to get the corresponding parameter estimates and calculate the differences. Alternatively, in BENCH we estimate $P(\hat{\Delta v} \mid y, \Delta y)$, the posterior probability for the pattern of change $\hat{\Delta v}$ conditioned on the observations $y$ and $\Delta y$. Using Bayes' rule:

$$
P(\hat{\Delta v} \mid y, \Delta y)=\frac{P(\Delta y \mid y, \hat{\Delta v}) P(\hat{\Delta v} \mid y)}{\sum_{\hat{\Delta v^{\prime}}} P\left(\Delta y \mid y, \hat{\Delta v}^{\prime}\right) P\left({\hat{\Delta v^{\prime}}}^{\prime} \mid y\right)}
$$

We assume no prior preference between the patterns of change (i.e. $P(\hat{\Delta v} \mid y)$ is uniform), so to estimate the posterior probability distribution we only need to estimate the likelihood term $P(\Delta y \mid y, \hat{\Delta v})$. The pattern of change $\hat{\Delta v}$ represents the direction but not the amount of the change in the parameters. We therefore marginalize the likelihood with respect to the amount of change ( $|\Delta v|):$

$$
P(\Delta y \mid y, \hat{\Delta v})=\int P(|\Delta v|) P(\Delta y|y, \hat{\Delta v},| \Delta v \mid) d|\Delta v|
$$

We assume that the prior distribution for the amount of change follows a log-normal pdf with a fixed mean and scale parameter (adjustable hyper parameters). A log-normal PDF is chosen to allow for changes across several order of magnitudes.

The likelihood term inside the integral, $P(\Delta y|y, \hat{\Delta v},| \Delta v \mid)$, defines how the measurements change as a result of a fully characterised vector of change in the parameters with the given direction $(\hat{\Delta v})$ and amount $(|\Delta v|)$. To relate this parameter change to a change in data one also needs to know the baseline parameters $(v)$, as

$$
\Delta y=\mathcal{M}(v+|\Delta v| \hat{\Delta v})-\mathcal{M}(v)+\epsilon
$$

where $\epsilon$ is the measurement noise. However, for a degenerate biophysical model, we cannot estimate a unique set of baseline parameters $v$ for which to estimate equation 3 . While, one could integrate over all possible values of $v$, this is a very high-dimensional integral, which would be very 
computationally expensive. Instead, we propose an alternative way to avoid the need of estimating the baseline parameters to estimate the likelihood.

Assuming that $|\Delta v|$ is reasonably small, and $\mathcal{M}$ is behaving smoothly w.r.t $v$, using a Taylor expansion we can express $\Delta y$ as :

$$
\Delta y=\nabla_{\hat{\Delta v}} \mathcal{M}(v)|\Delta v|+\epsilon
$$

Where $\nabla_{\hat{\Delta v}} \mathcal{M}(v)$ is the gradient of $\mathcal{M}$ in the direction of $\hat{\Delta v}$ at point $v$, and $\epsilon$ is the measurement noise. Given the baseline measurements $(y)$, but not the baseline parameters $(v)$, there can be an infinite number of $\nabla_{\hat{\Delta v}} \mathcal{M}(v)$ for a degenerate model (Figure 2). To account for all instances of the gradient, we model $\nabla_{\hat{\Delta v}} \mathcal{M}$ given $y$ as a random variable that follows a normal distribution with hyperparameters $\mu(y)$ and $\Sigma(y)$, i.e.

$$
P\left(\nabla_{\hat{\Delta v}} \mathcal{M} \mid y\right)=N\left(\nabla_{\hat{\Delta v}} \mathcal{M} ; \mu_{\hat{\Delta v}}(y), \Sigma_{\hat{\Delta v}}(y)\right)
$$

where $\mu_{\hat{\Delta v}}$ represents the average expected change in the measurements as a result of change in parameters in the direction $\hat{\Delta v}, \Sigma_{\hat{\Delta v}}$ represents the uncertainty around this expectation due to the unknown baseline parameters (Figure 2), and $N(x ; m, C)$ represents a Gaussian PDF with mean $m$ and covariance $C$ evaluated at point $x$. This allows us to transfer the uncertainty in the baseline parameters to an uncertainty in the measurement space, which we can model and predict. In the next section we will describe a method for estimating these hyperparameters from the measurements by training regression models on simulated data. Once we compute these hyperparameters, by inserting equation 5 back into equation 4 we can compute the likelihood term inside the integral as

$$
P(\Delta y|y, \hat{\Delta v},| \Delta v \mid)=N\left(|\Delta v| \mu_{\hat{\Delta v}},|\Delta v|^{2} \Sigma_{\hat{\Delta v}}+\Sigma_{n}\right)
$$

where $\Sigma_{n}$ is the noise covariance matrix.

Finally, by computing the integral over the size of the parameter change in equation 2 numeri- 
Parameter Space

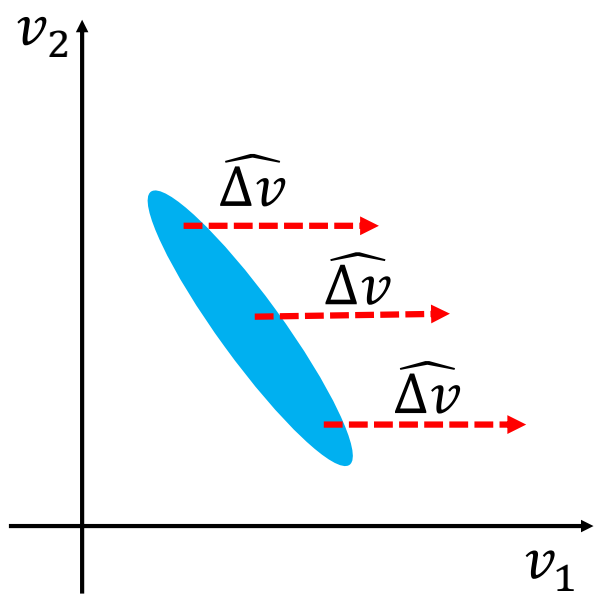

Measurement Space

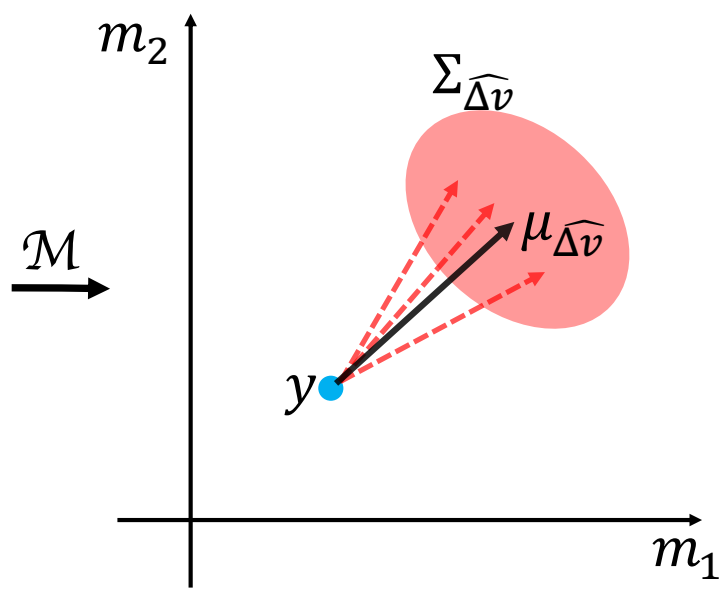

Figure 2. Distribution of gradients. The way measurements change as a result of a particular change in the parameters can only be calculated if we know the baseline parameters. When we are only given the measurements, there are several instances of equally likely gradient directions depending on the underlying baseline parameters. We model all of these gradients given the baseline measurements as a random variable with a presumed distribution. This allows us to transfer the uncertainty due to the inverse model degeneracy into the measurement space. The blue oval in the parameter space (left) represents all the parameter settings that map onto the same blue point in measurement space(right). Each of these parameter settings can produce a different gradient direction in the measurements space. The collection of such gradients of change $\hat{\Delta v}$ for the measurement $y$ are modelled as a Gaussian distribution with mean $\mu_{\hat{\Delta v}}(y)$ and covariance $\Sigma_{\hat{\Delta v}}(y)$.

cally, we are able to approximate the likelihood function $P(\Delta y \mid y, \hat{\Delta v})$ which we can then use in equation (1) yielding the desired posterior distribution on the change in parameters.

Moreover, using the approximation of the likelihood function in equation 6 the posterior probability of the amount of change in each parameter is proportional to

$$
P(|\Delta v| \mid \Delta y, y, \hat{\Delta v}) \propto P(\Delta y|y, \hat{\Delta v},| \Delta v \mid) P(|\Delta v|)
$$

Accordingly, we can estimate the most likely amount of change in the parameter given the measurements by finding the $|\Delta v|$ that maximizes the above posterior probability (maximum a posteriori estimation). Alternatively, we can estimate the expected value of the amount of change by integrating this posterior probability distribution multiplied by $|\Delta v|$ over $|\Delta v|$. 


\section{Training models of change}

In this section we describe how to train a regression model to estimate the hyperparameters of the distribution of $\nabla_{\hat{\Delta v}} \mathcal{M}(v)$, namely the average $\left(\mu_{\hat{\Delta v}}(y)\right)$ and uncertainty $\left(\Sigma_{\hat{\Delta v}}(y)\right)$ of change in the measurement $(y)$ for a parameter change $(\hat{\Delta v})$.

Given some baseline parameters ( $v$ ) we can calculate the baseline measurements as $y=\mathcal{M}(v)$ and approximate the gradient in direction $\hat{\Delta v}$ using

$$
\nabla_{\hat{\Delta v}} \mathcal{M}(v) \approx \lim _{t \rightarrow 0} \frac{\mathcal{M}(v+t \hat{\Delta v})-\mathcal{M}(v)}{t}
$$

Therefore, by sampling $v$ from the parameter space using a prior distribution, we generate a simulated dataset of pairs $\left[y, \nabla_{\hat{\Delta v}} \mathcal{M}\right]$ that we use for training regression models.

We use a regression model parameterised by $w_{\mu_{\hat{\Delta v}}}$ to estimate $\mu_{\hat{\Delta v}}$ as:

$$
\mu_{\hat{\Delta v}}\left(y ; w_{\mu_{\hat{\Delta v}}}\right)=F(y) \cdot w_{\mu_{\hat{\Delta v}}}
$$

where $F(y)$ is the design matrix, which depends on arbitrary affine or non-linear transformations of $y$. Note that the subscript $\mu_{\hat{\Delta v}}$ of the weights indicates that each pattern of change in the parameters has its own set of weights.

We also employ a regression model for the uncertainty hyperparameter $\Sigma_{\hat{\Delta} v}$ parameterised by $w_{\Sigma_{\hat{\Delta v}}}$. However, $\Sigma_{\hat{\Delta v}}$ must be positive definite, which would not be guaranteed when directly estimating $\Sigma_{\hat{\Delta v}}$ by training an element-wise regression model. To account for the positive definite nature of $\Sigma_{\hat{\Delta} v}$, we instead train regression models for elements of the lower triangular matrix of its Cholesky decomposition $(L)$. Also, since the diagonal elements of the lower-triangular matrix in Cholesky decomposition must be non-negative, we use their log-transform in the regression. Hence

$$
\Sigma_{\hat{\Delta v}}\left(y ; w_{\Sigma_{\hat{\Delta v}}}\right)=\mathcal{T}\left(F(y) \cdot w_{\Sigma_{\hat{\Delta v}}}\right)
$$

where $\mathcal{T}$ is the transformation of the regressed vector to the full covariance matrix that includes 
the arrangement of elements, exponentiation of the diagonals, and matrix multiplication.

Putting back the above regression models into equation 5 the likelihood of observing pairs of baseline measurements and gradients in terms of the parameters of regression models is:

$$
L\left(w_{\mu_{\hat{\Delta v}}}, w_{\Sigma_{\hat{\Delta v}}}\right)=\prod_{i} N\left(\nabla_{\hat{\Delta v}} \mathcal{M}_{i} ; F\left(y_{i}\right) \cdot w_{\mu_{\hat{\Delta v}}}, \mathcal{T}\left(F\left(y_{i}\right) \cdot w_{\Sigma_{\hat{\Delta v}}}\right)\right)
$$

Accordingly, we estimate the optimal weights $w_{\mu_{\hat{\Delta v}}}, w_{\Sigma_{\hat{\Delta v}}}$ by maximizing the above likelihood function for the simulated dataset using using a combination of the BFGS and Nelder-Mead methods as implemented in SciPy (Virtanen et al. 2020).

This procedure is repeated for each hypothetical pattern of change, yielding two sets of weights for the average and uncertainty of change, which we refer to as a " change model". Once we estimated these weights, for any given baseline measurement we use the regression models in equations 9 and 10 to estimate the distribution of gradients. Figure 3 shows a schematic overview of the inputs, outputs and steps that are required to train a change model, as well as how to use them to infer the change in parameters.

In this work, we used a second degree polynomial function of the data for the regression models that estimate the mean change $\left(\mu_{\hat{\Delta v}}\right)$ from the baseline measurements. For the uncertainty parameter $\left(\Sigma_{\hat{\Delta v}}\right)$ a first degree (linear) model is chosen as we expect less variability across samples for this parameter. The weights for the regression models were estimated using a maximum likelihood optimization and a training dataset with 100,000 simulated samples.

\section{Biophysical model of diffusion}

In this section we explain the biophysical model of diffusion that we used to model brain microstructure with diffusion MRI data. The diffusion signal $S$ in the brain is conventionally modelled as the sum of signals from multiple compartments. We will here adopt the threecompartment standard model (Novikov et al. 2019a) consisting of an isotropic free water (denoted 


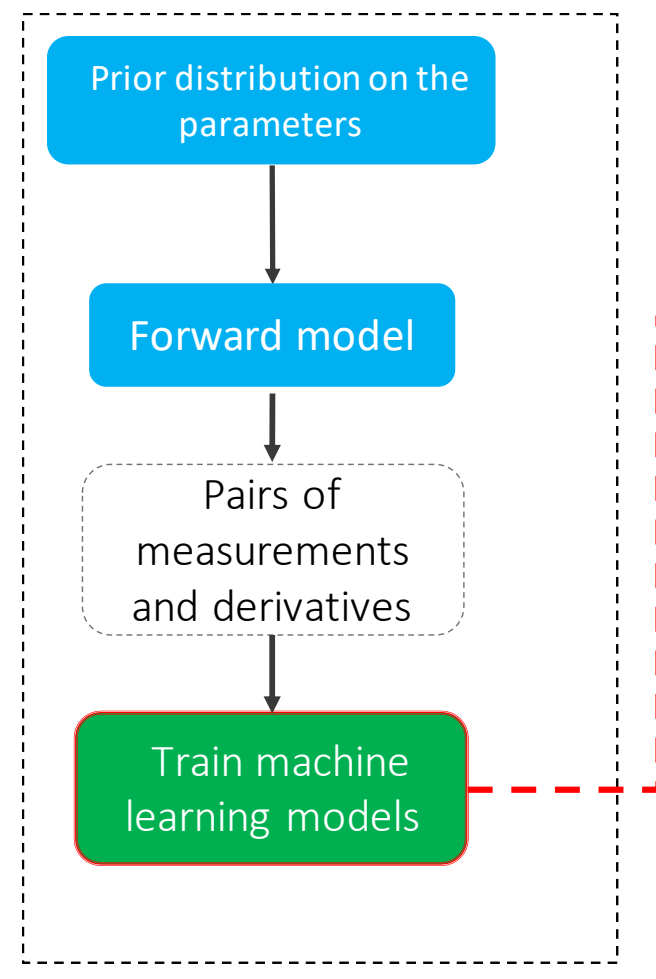

a) Training

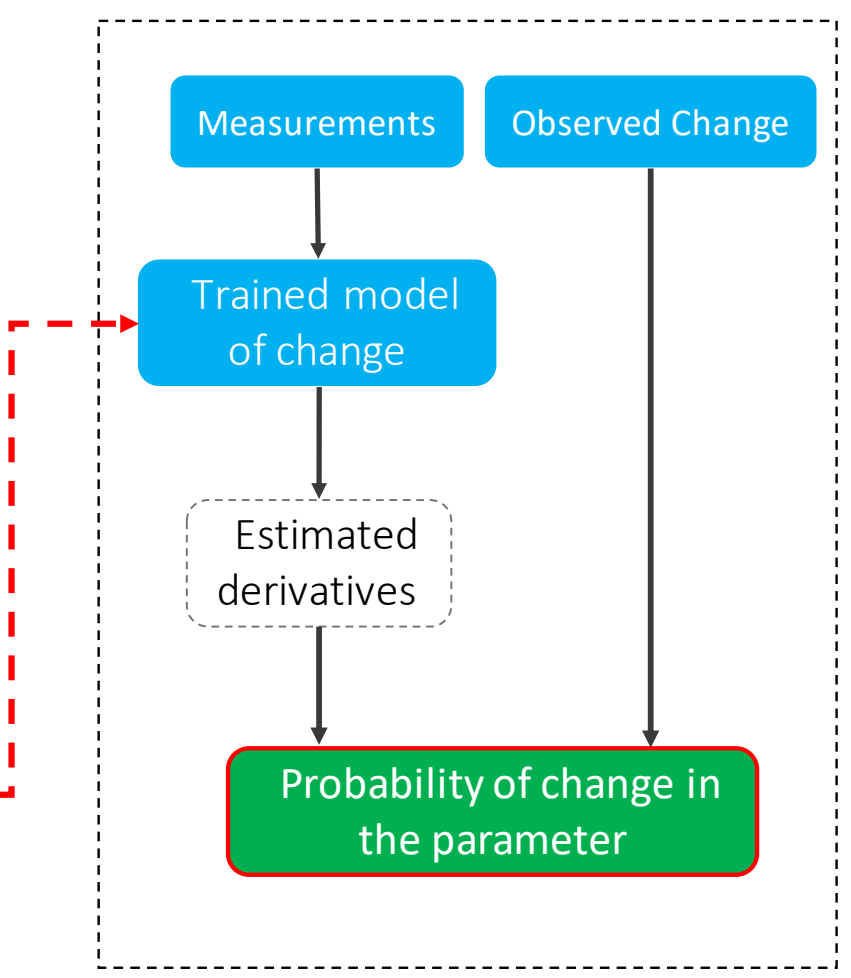

b) Inference

Figure 3. Schematic flowchart for training inference of change models. The blue, white and green blocks indicate user defined inputs, intermediate variables and outputs respectively. In the training phase for each parameter change, samples that are drawn from the provided prior distribution are passed through the forward model to estimate pairs of measurements and derivatives. Then, regression models are trained to estimate the distribution of derivatives given the measurements using a maximum likelihood estimation. This phase does not require real data and needs to be done only once. In the inference stage using these trained models we estimate the distribution of the derivatives for any given baseline measurements. We then calculate the posterior probability that change in each parameter caused the change in the measurements using the derivative distributions.

by the subscript "iso"), an intra-axonal ( "in"), and an extra-axonal ( "ex") compartment:

$$
S=S_{i s o} A_{i s o}+S_{i n} A_{i n}+S_{e x} A_{e x}
$$

where $S_{i}$ represents the baseline signal contribution (at $b=0$ ), and $A_{i}$ represents the signal attenuation due to the diffusion weighting in each compartment (Figure 4).

The attenuation for the isotropic compartment is modelled as an exponential decay:

$$
A_{i s o}=e^{-b d_{i s o}}
$$


where $d_{i s o}$ is the diffusion coefficient of free water.

The intra-axonal compartment is modelled as a set of dispersed identical sticks with no perpendicular diffusivity. Therefore, the signal attenuation for this compartment is the spherical convolution of a stick response function with an orientation distribution function. The stick response function for gradient direction $g$ and $\mathrm{b}$-value $b$ is given by

$$
R\left(b, g ; \mu, d_{i n, a}\right)=e^{-b d_{i n, a}\left(\mu^{T} g\right)^{2}}
$$

where $d_{i n, a}$ is the diffusion coefficient along the orientation of the stick $\mu$. The fibre Orientation Distribution Function (fODF) is modelled with a Watson distribution, which is defined as

$$
f(x)=\frac{1}{c} e^{\kappa\left(\mu^{T} x\right)^{2}}
$$

where $\mu$ is the average orientation, $\kappa$ is the concentration coefficient and $c$ is a normalization constant. To assimilate the dispersion coefficient to the notion of variance and limit it to a bounded range, we use the change of variable from $\kappa$ to Orientation Dispersion Index (ODI) as $O D I=\frac{2}{\pi} \arctan \left(\frac{1}{\kappa}\right)$. Unlike $\kappa$ which is unbounded, $O D I$ is limited to the range $(0,1)$, where higher $O D I$ values correspond to more dispersion. The diffusion signal for this compartment is the spherical convolution of the fiber response function with the Watson ODF:

$$
A_{i n}=\iint_{S^{2}} e^{-b d_{i n, a}\left(g^{T} n\right)^{2}} \frac{1}{c} e^{\frac{2}{\pi} \tan ^{-1}(O D I)\left(\mu^{T} n\right)^{2}} d n
$$

where the integral is over the surface of the unit sphere $S^{2}$ representing all possible fibre orientations in 3D.

The extra-axonal compartment is modelled similar to the intra-axonal compartment, with the addition of a non-zero diffusion perpendicular to the fiber orientation. The fiber response function in this case is given by

$$
R=e^{-b\left[d_{e x, a}\left(\mu^{T} g\right)^{2}+d_{e x, r}\left(1-\left(\mu^{T} g\right)^{2}\right)\right]}
$$



their valid range.

\begin{tabular}{clc}
\hline \hline Parameter & \multicolumn{1}{c}{ Description } & Range \\
\hline$s_{i s o}$ & Signal fraction for isotropic (free water) diffusion compartment & {$[0,1]$} \\
\hline$s_{i n}$ & Signal fraction for intra-axonal compartment & {$[0,1]$} \\
\hline$s_{e x}$ & Signal fraction for extra-axonal compartment & {$[0,1]$} \\
\hline$d_{i s o}$ & Isotropic (free water) diffusivity coefficient & {$[0, \infty]$} \\
\hline$d_{i n, a}$ & Parallel diffusivity for the intra-axonal compartment & {$[0, \infty]$} \\
\hline$d_{e x, a}$ & Parallel diffusivity for the extra-axonal compartment & {$[0, \infty]$} \\
\hline$\tau$ & radial to axial diffusivity ratio for the extra-axonal compartment & {$[0,1]$} \\
\hline$O D I$ & Orientation dispersion index & {$[0,1)$} \\
\hline \hline
\end{tabular}

TABLE 1. Microstructural parameters of the diffusion model. All diffusion coefficients are in $\mu \mathrm{m}^{2} / \mathrm{ms}$

As the compartments share the same geometry, the same fibre orientation distribution is used. Accordingly, the signal attenuation for extra-axonal compartment is given by

$$
A_{e x}=\iint_{S^{2}} e^{-b d_{e x, a}\left[\left(\mu^{T} g\right)^{2}+\tau\left(1-\left(\mu^{T} g\right)^{2}\right)\right]} \frac{1}{c} e^{\frac{2}{\pi} \tan ^{-1}(O D I)\left(\mu^{T} n\right)^{2}} d n
$$

We use the confluent hypergeometric function of the first kind with matrix argument to compute the integrals for both intra and extra axonal compartments similar to (Sotiropoulos et al. 2012).

Table 1 reports all the free parameters of the described biophysical diffusion model along with 


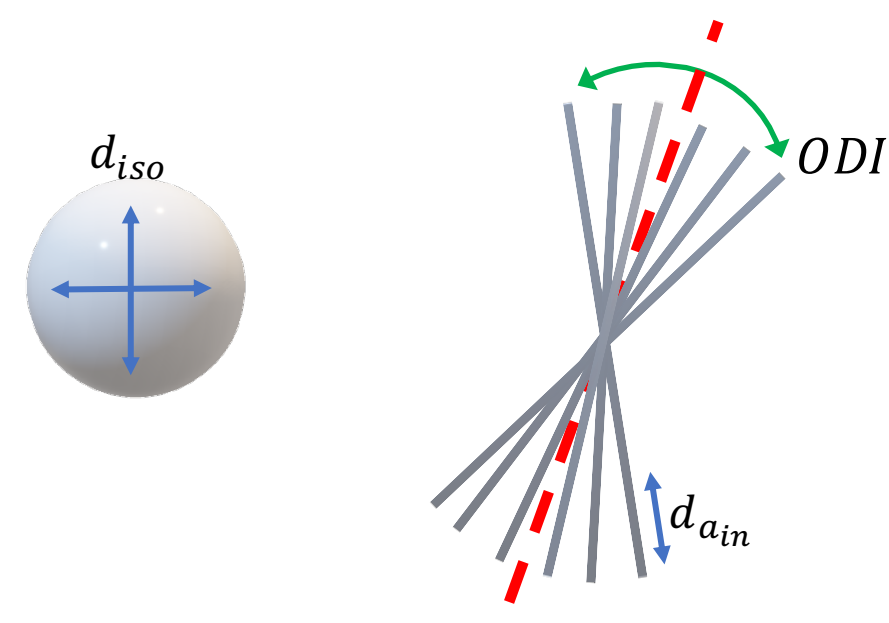

Isotropic
Intra-axonal

Figure 4. Compartments of the diffusion model. We use a three compartment model that can model diffusion MRI signals from various brain tissues namely CSF, white matter and gray matter. The isotropic compartment models unrestricted diffusion of water molecules outside of tissue (CSF) with a single free parameter $d_{i s o}$. The intra-axonal compartment models the diffusion of water within axons as several sticks with the same parallel diffusivity parameter $d_{i n, a}$, with zero radial diffusivity, that are dispersed by a Watson distribution with orientation dispersion index $O D I$. The extra-axonal compartment is also a Watson dispersed stick model with parallel diffusivity $d_{e x, a}$ and perpendicular diffusivity $d_{e x, r}=\tau d_{e x, a}$. Including the signal fraction parameters $\left(s_{i s o}, s_{i n}, s_{e x}\right)$ this model has 8 free parameters, which are more than that can be fitted to a conventional dMRI data.

\section{Summary measurements}

Diffusion MRI data are usually measured in multiple shells to capture tissue properties that are sensitive to diffusion of water molecules at various spatial scales. Within each shell, gradients are applied in several directions to measure the geometrical structure of the tissue. However, since we are only interested in the microstructural characteristics, any orientation-related information is irrelevant. We therefore need summary measurements from each shell that are invariant to orientations. We create these summary measurements using real spherical harmonics, which are analogous to the Fourier transform for the spherical domain.

Spherical harmonics are a complete set of orthonormal functions over the surface of a unit sphere. That is to say, any bounded real function that is defined over the unit sphere can be represented by a unique linear combination of these functions with real coefficients. Each real spherical harmonic is denoted by $Y_{l, m}(\theta, \phi)$ where $l=0,1,2, \ldots$ is the degree and $m=-l, \ldots, l$ is 
the order, and $\theta \in[0, \pi], \phi \in[-\pi, \pi]$ are the polar and longitudinal angles in standard spherical coordinate system respectively. The diffusion signal at each shell is decomposed as:

$$
S(\theta, \phi)=\sum_{l=0}^{\infty} \sum_{m=-l}^{l} C_{l, m} Y_{l, m}(\theta, \phi)
$$

Since the harmonics are a linear basis, one can easily calculate the coefficients for the signal in each shell by inverting the design matrix formed by the harmonics sampled at the gradient directions.

The coefficients are not orientationally invariant. However, the total power in each degree, which is defined as the vector norm of all the coefficients in that degree, is rotationally invariant (Kazhdan et al. 2003; Zucchelli et al. 2020; Novikova et al. 2018). Also, since the diffusion signal is symmetric around the origin and the harmonics of odd degree are odd functions (anti-symmetric w.r.t origin), all odd degrees have zero coefficients.

Consequently, for each shell of diffusion data, we calculate the mean squares of all coefficients for degrees $l=0,2,4, \ldots$ as the orientationally-invariant summary measurements.

$$
y_{l}=\frac{1}{2 l+1} \sum_{m=-l}^{l} C_{l, m}^{2}
$$

The mean is chosen over the norm to make the scale equal across all degrees. For the case of $l=0$, we simply use the only coefficient (without the square), so that it represents the mean signal. The higher order summary measurements quantify the signal anisotropy; with greater $l$ being more sensitive to sharper changes. We used a logarithm transformation on the power of the coefficients to make the distribution across the brain closer to a Gaussian and more sensitive to smaller changes.

\section{METHODS}

\section{Simulations}

For all the simulations we used the acquisition protocol conducted by the UK Biobank (UKB) (Miller et al. 2016; Alfaro-Almagro et al. 2018) which includes two shells of diffusion $\left(b=1,2 \frac{\mathrm{ms}}{\mu \mathrm{m}^{2}}\right)$ with linear diffusion encoding. Each shell consists of 50 gradient directions distributed uniformly 
over the surface of the unit sphere, in addition to 5 acquisitions with $b=0$, yielding a total of 105 measurements.

We used the rotationally invariant summary measurements computed from spherical harmonics for signal representation. The summary measurements for each shell are norms of coefficients at $l=0$ (absolute value) and $l=2$ (log mean squared). This produces 5 rotational invariant summary measurements from a diffusion data, namely b0-mean, b1-mean, b1-l2, b2-mean, and b2-l2.

The described standard model for diffusion is used for both simulated test data and for training models of change. The prior distributions for the parameters are shown in figure 5 . We note that these priors are not used for constraining the model parameters but rather they are used to generate training samples for the regression models. The choice of the prior distributions is arbitrary as long as they can reflect all hypothetical parameter combinations that can produce measurements similar to real data.

The standard model is not invertible given a conventional multishell diffusion data with linear diffusion encoding (Novikov et al. 2019a; Jelescu et al. 2016) . Typically, additional constraints are imposed to render the model invertible, e.g. in NODDI (Zhang et al. 2012), the diffusion coefficients are fixed to a prior value as follows:

$$
d_{i s o}=3 \frac{\mu m^{2}}{m s}, d_{i n, a}=d_{e x, a}=1.7 \frac{\mu m^{2}}{m s}
$$

Additionally, the tortuosity parameter $\tau$ is coupled to the signal fractions:

$$
\tau=\frac{s_{\text {in }}}{s_{\text {in }}+s_{\text {ex }}}
$$

Accordingly, this constrained model has four free parameters: $s_{i s o}, s_{i n}, s_{e x}$ and $O D I$.

For both the constrained and unconstrained models, we generated a test dataset containing pairs of simulated diffusion signals, such that in each pair at most one microstructural parameter is different. To generate each pair, we sample a baseline parameter setting from the prior distributions and change one of the parameters by an effect size of 0.1 . We also generate pairs of data where no 

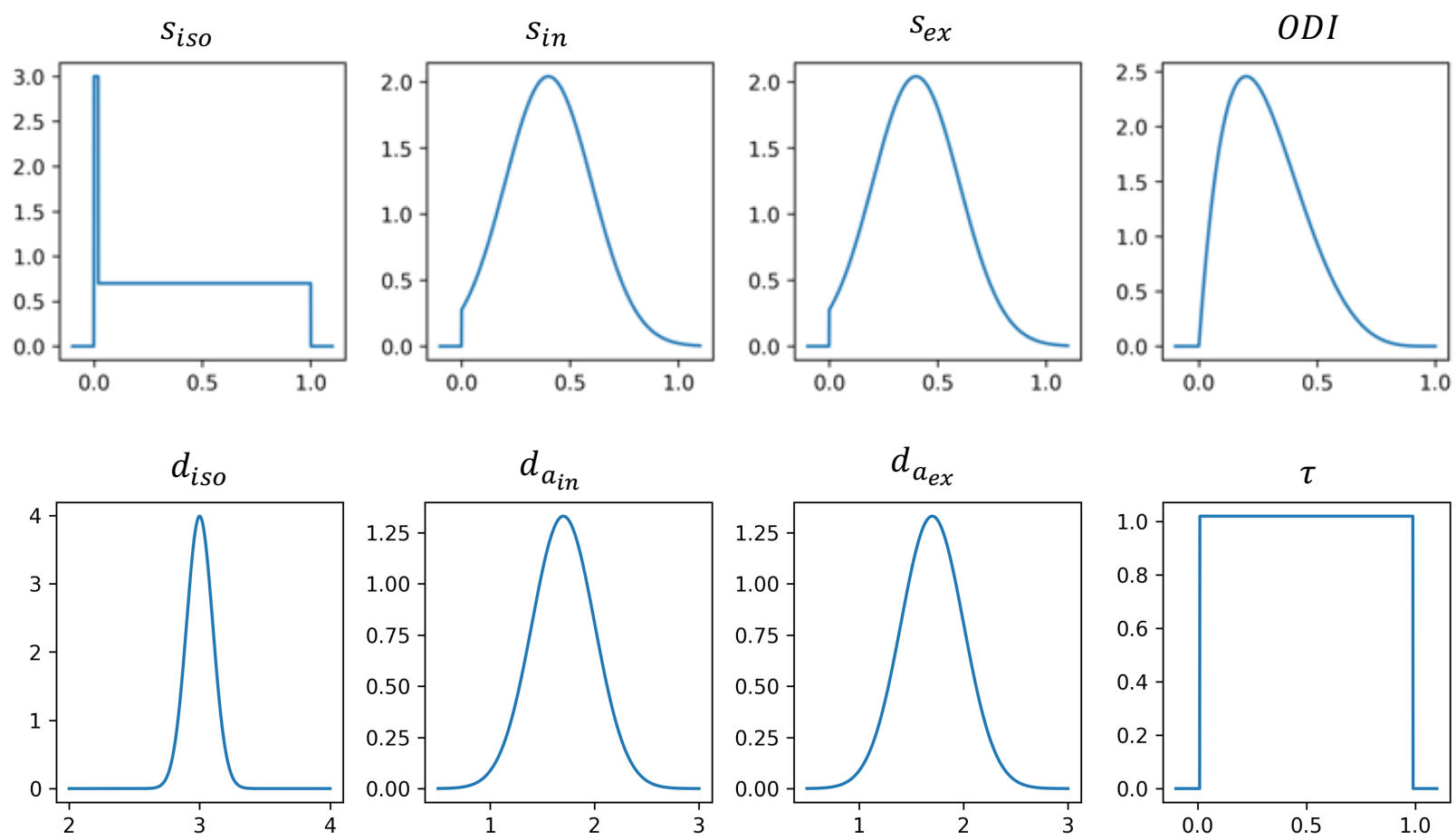

Figure 5. Prior distributions for the parameters of the standard model. These priors are used for generating pairs of measurements and gradients for training the models of change. Also, the same priors are used for simulating test datasets. The priors are chosen such that they contain all probable parameter combinations that can produce measurements similar to real data. The delta function along with uniform distribution in the isotropic signal fraction is used to model pure tissue types as well as partial volume effect. In the training phase, the volume fractions are normalized to sum up to 1 . A beta (shape parameters $\alpha=2, \beta=5$ ) distribution is used for $O D I$ to impose a uniform distribution for effective fibre dispersion. The prior for isotropic and axial diffusivities are normal distributions with mean 3 and $1.7\left(\frac{\mu m^{2}}{m s}\right)$ and standard deviation 0.1 and 0.3 respectively; as we expect faster diffusion as well as less variability in the free water component.

parameter changes and the difference between the two samples is only due to the addition of noise.

We then apply the forward model to both parameter settings to produce diffusion MRI signals. Gaussian noise with standard deviation $\sigma_{n}=0.02(\mathrm{SNR}=50)$ is added to all diffusion signals.

The signal fraction parameters are constrained to sum up to 1 for training models of change. Note that whilst this imposes a constraint that the $b 0$-mean for the baseline measurement is equal to 1 , it does not constrain a change in that summary measurement. Accordingly, all the summary measurements (both in the baseline and the change vector) are normalized by the b0-mean of the baseline measurement for any real data. This differs from the conventional NODDI, where there is a constraint on the signal fractions to sum up to 1 for each sample, which reduces the free parameters 
to 3 , but here we assume all the parameters can change independently.

For the direct inversion approach, a maximum a-posteriori algorithm is employed to estimate the parameters of the constrained model from each diffusion signal separately. Then using a z-test across the parameter estimates in each pair, we calculate a p-value for the change in each parameter (corrected for multiple comparisons across parameters). The parameter with the minimum p-value is identified as the changed parameter. All the cases with minimum $p>0.05$ are identified as no change.

We also used BENCH for identifying change on the same dataset. To estimate the noise covariance in the summary measurements $\Sigma_{n}, 100$ noisy instances of signals were generated, and the sample covariance of the difference between summary measurements in each pair was estimated. We then estimated the posterior probability of change in each parameter using the trained models of change. The no change model has a zero mean and covariance $\Sigma_{n}$ everywhere. The change model with the maximum posterior probability is selected as the predicted change.

\section{White matter hyperintensities}

We employed the trained models of for the parameters of the standard model to investigate the microstructural changes in white matter hyperintensities (WMH). In this experiment, we used diffusion MRI of 2400 randomly selected subjects from the UK biobank dataset. To account for the variability in overall intensity across subjects, we divided each subject's diffusion data by the average intensity of the $b 0$ image across the brain's white and grey matter extracted using FSL FAST (Zhang et al. 2000). We then computed the spherical harmonics-based summary measurements from the diffusion MRI data for each subject and interpolated these measures into the standard MNI space using non-linear transformations estimated by FSL FNIRT (Woolrich et al. 2009; Andersson et al. 2019).

Segmentations of the WMHs were generated from T2 FLAIR images using FSL's BIANCA (Griffanti et al. 2016) as part of the UK Biobank pipeline (Miller et al. 2016). We computed the average summary measurements for normally appearing white matter (voxels within the white matter mask not classified as WMH) and the WMHs for all voxels that included more than 10 
subjects with WMH. For each voxel, subjects were split into two groups according to whether the voxel has been classified as WMH or not. Averaging the summary measures within groups provides us with the baseline measurement $(y)$ and the observed change $(\Delta y)$ related to $\mathrm{WMH}$. The noise covariance $\left(\Sigma_{n}\right)$ in each voxel was estimated using the within group covariance matrix divided by the number of subjects in the normal appearing white matter group.

\section{RESULTS}

\section{Summary measures}

A representative axial slice of the normalized summary measurements from a single subject are shown in Figure 6. The "mean" summary measures represent the normalised average signal. The $l 2$ measures quantify the anisotropy in each voxel (similar to Fractional Anisotropy maps in DTI).

The bottom panels of Figure 6 show histograms of the summary measurements across the brain for the same subject, as well as distributions of simulated data based on prior distributions over the model parameters. The distribution for the generated samples fully covers the range of the data and follows the same density distribution. This verifies that the prior distributions are wide enough to capture the full range of real data.

Figure 7 shows estimated derivatives of the summary measurements at baseline data representative of putative voxels in the white matter and grey matter. The error bars show estimated standard deviations of the derivatives (the square root of diagonals of the estimated covariance matrix). This variance is reflecting the uncertainty in the underlying parameters that can generate these measurements, as well as residuals of the regression model for the mean.

\section{Simulations}

We first employed simulated data to evaluate the performance of the proposed approach in inferring microstructural changes from diffusion MRI data.

\section{Comparison with model inversion}

Figure 8 shows the confusion matrix using model inversion (left), and our inversion-free approach (right) for an invertible model with only 4 free parameters. Each element of these matrices 

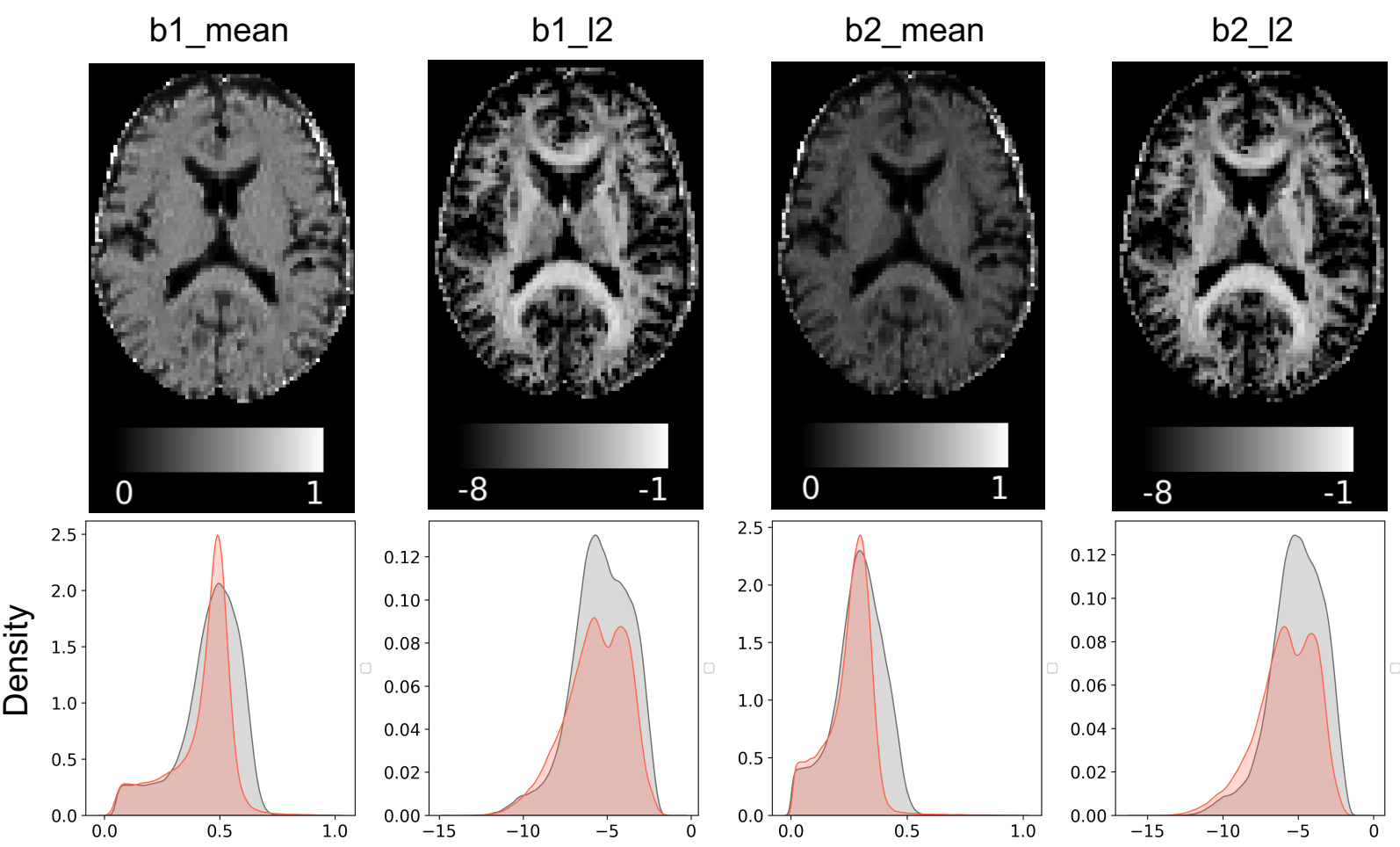

Figure 6. Maps of the summary measurements for a sample subject in the UK biobank dataset (top) and their histogram (bottom). The mean summary measurements is reflecting the mean diffusivity in each shell. The 12 summary measurements estimate the anisotropy, which is similar to the fractional anisotropy (FA), but computed with a linear transformation of the signal. Histograms show the distribution of these measurements across the brain; as well as the distribution of simulated data using the standard model and provided prior distributions. This shows that the simulations capture the full range of the summary measures from real data.

represents the percentage of times a change in the parameter represented at the corresponding column is identified as a change in the corresponding row. Both approaches were able to detect the true change in most of the cases.

For the standard model with all 8 free parameters, Figure 9 shows the confusion matrices using the direct model inversion (left) and change estimation (right). Since the uncertainties of the parameter estimates are very large due to the model degeneracies, almost all of the changes are confused with no change when using direct inversion. However, the inversion-free approach is able to identify changes in $s_{i s o}, s_{i n}, s_{e x}$ and $O D I$. Although, there is confusion between the remaining parameters compared to the restricted model, here we do not make any strong assumptions on the value of those parameters. Also, most of the confusions for these parameters are between them, meaning that we are able to distinguish a change in those parameters (e.g. the diffusivity 


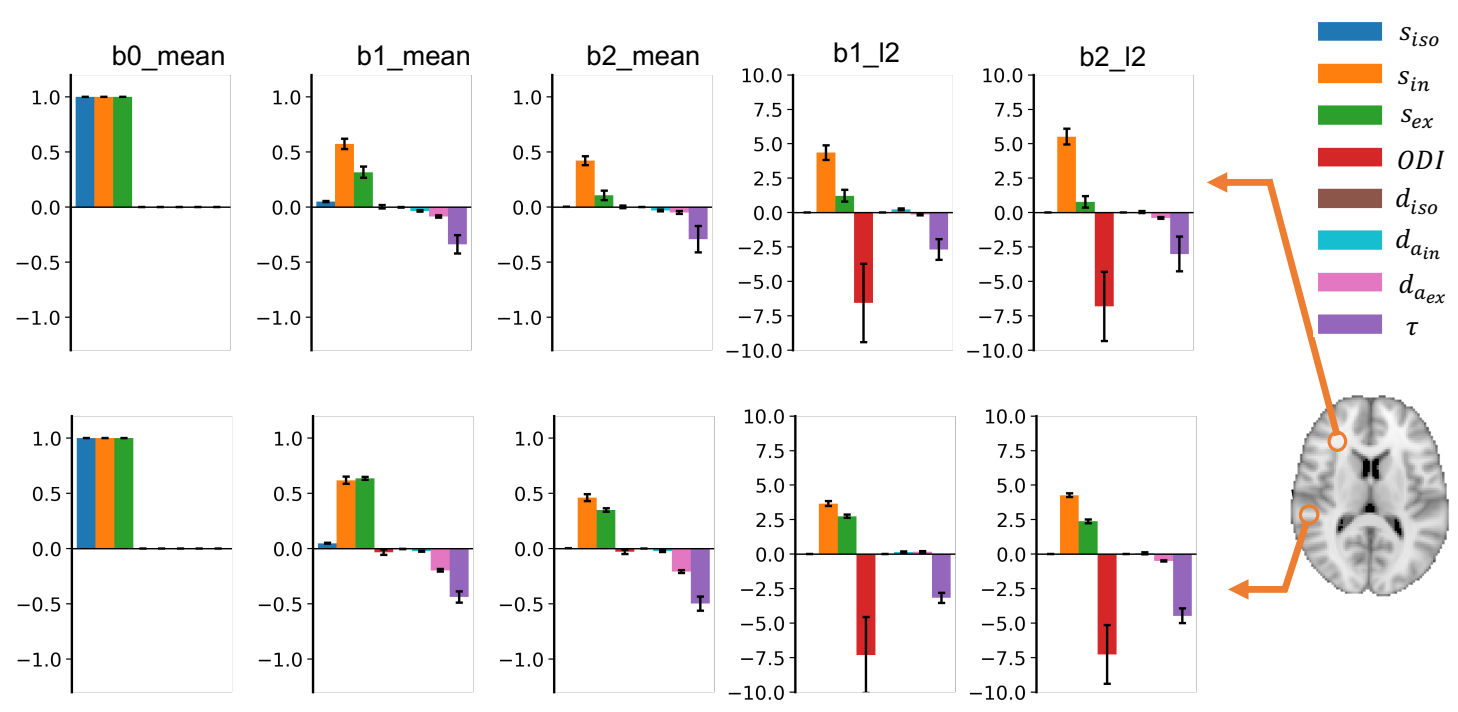

Figure 7. The estimated amount of change in the summary measurements as a result of a unit change in each parameter $\left(\mu_{\hat{\Delta v}}\right)$ for a sample white matter and grey matter voxel. The error bars show the estimated standard deviation of change. Colors correspond to parameters and columns indicate summary measurements. Due to differences in the baseline, each voxel can have a different change vector for the same parameter change. This added degree of freedom can model the variability of parameters (e.g. diffusivities) across the brain, which is not considered in restricted models; e.g. NODDI.
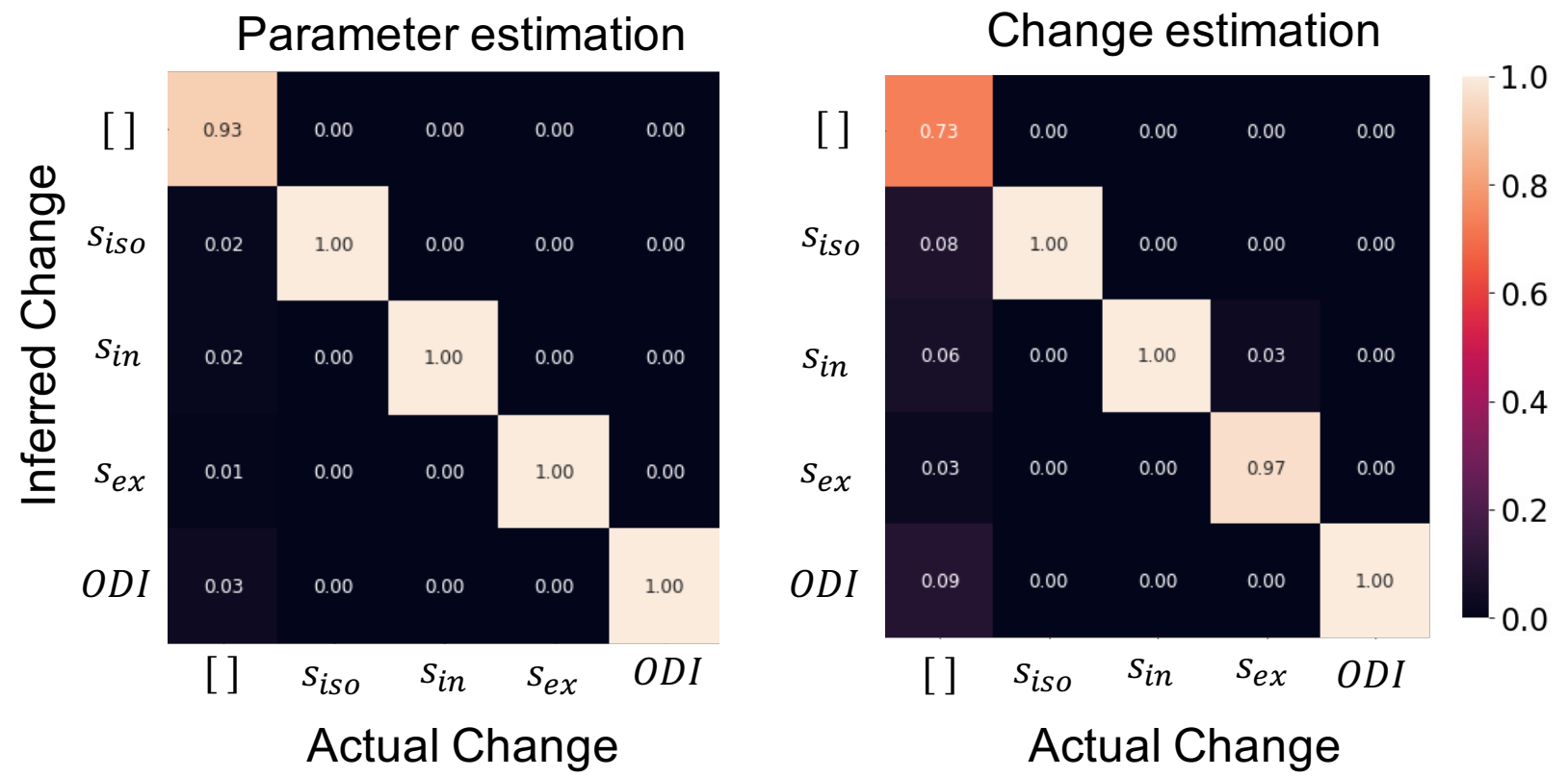

Figure 8. Confusion matrices for the constrained model using the direct inversion and the proposed approach. The numbers indicate the percentage of time a change in the corresponding column is identified as a change in the corresponding row for each approach. The diagonal elements show the accuracy in identifying true change. Both of the approaches performed near to ideal in detecting the true change. The change estimation has more false positives, but unlike the inversion approach, we did not explicitly define a false positive rate threshold. 
parameters) from others. Change in isotropic diffusivity is mostly confused with the no change model. This is due to the $b$-values in the UKB protocol which are too high for this parameter; a change in this parameter has minimal effect on the signal. The changes in parallel diffusivity parameters $d_{i n, a}, d_{e x, a}$ are mostly detected correctly or confused with one another. This is because, specifically at low tortuosity, these two parameters affect the diffusion signal in a similar way.
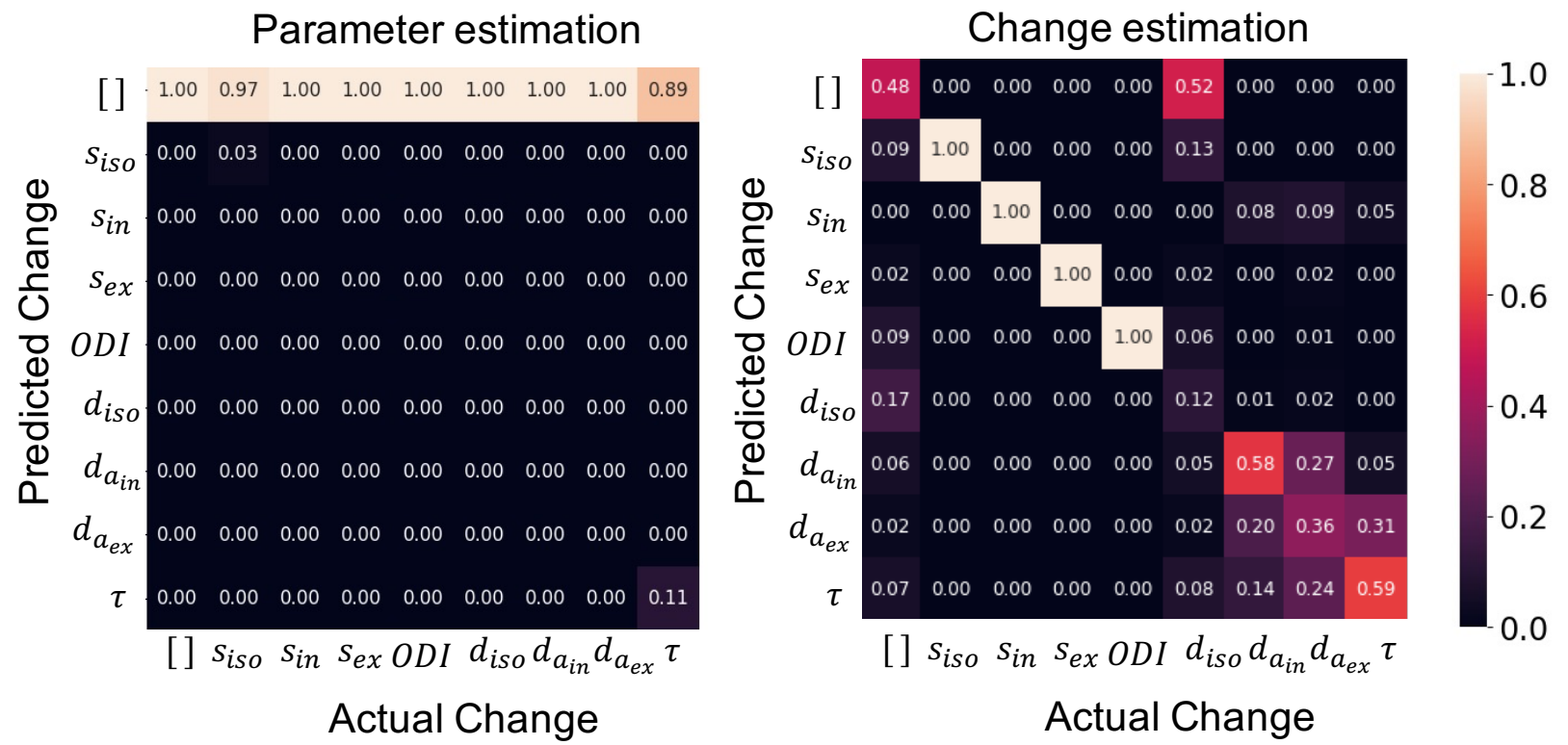

Figure 9. Confusion matrices for the full model using parameter estimation (left) and change estimation (right). Given diffusion data at few shells, the full model is not invertible, i.e. the parameter estimates have a high variance. Therefore, almost no significant change is detected using parameter estimates. On the other hand, the change estimation approach can still identify changes in all the parameters of the restricted model. Although there remains confusion between a subset of the parameters when these have similar effects on the diffusion signal

\section{Sensitivity to change in each parameters}

To evaluate the sensitivity of the approach to the amount of change in each parameter, we generated test datasets with variable effect sizes starting from 0 to 0.15 with step sizes of 0.01 . Figure 10 shows the average posterior probability of change in each parameter versus the effect size. In all types of change, at very small effect sizes $(<0.01)$ the change is confused with no change, but as the effect size increases the probability of identifying the true change (red curves) increases. Changes in all signal fraction parameters and in the fibre dispersion are identified with high accuracy even at very small effect sizes (note that these are the parameters allowed to vary 

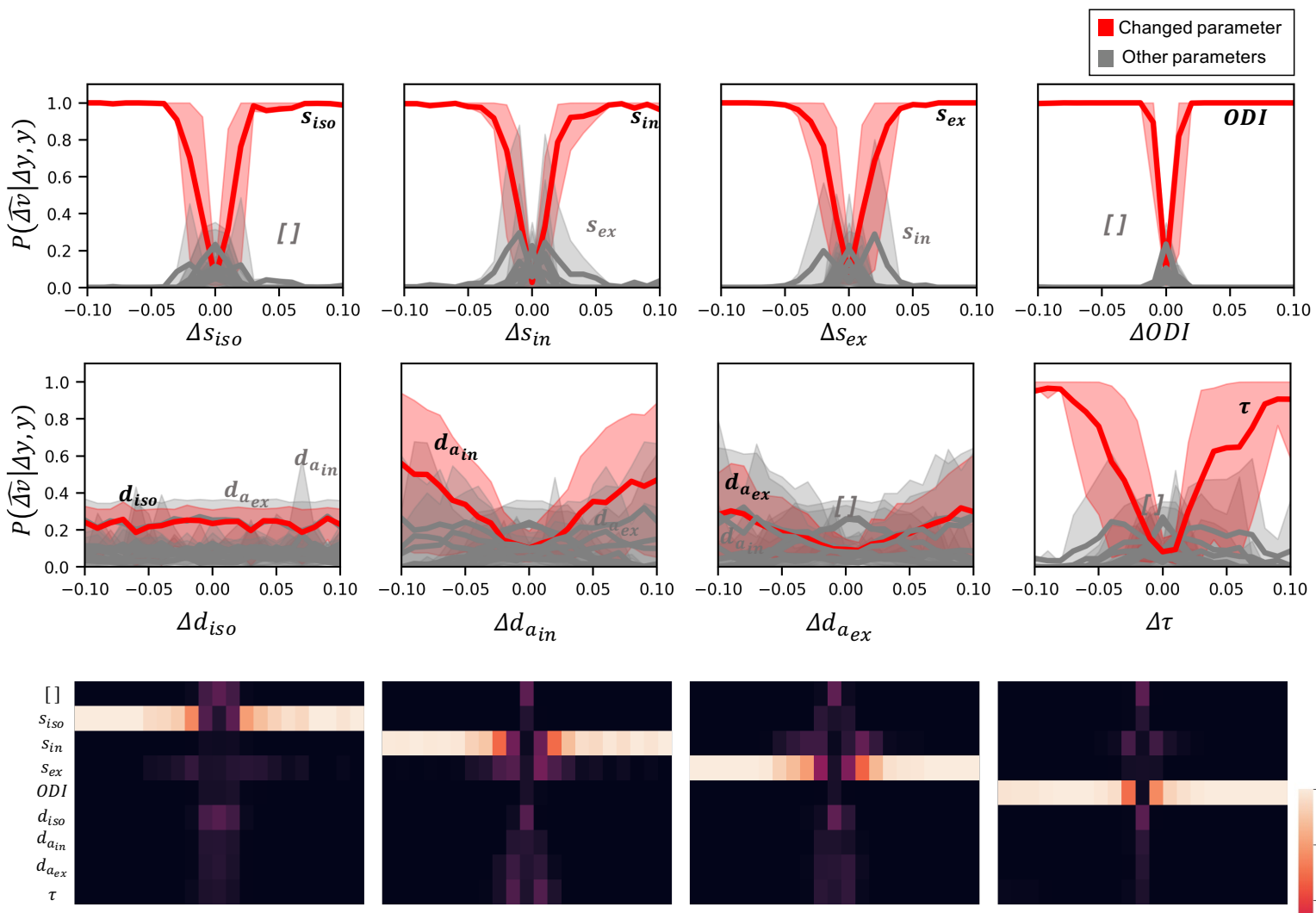

$\Delta s_{i s o}$

$\Delta s_{e x}$
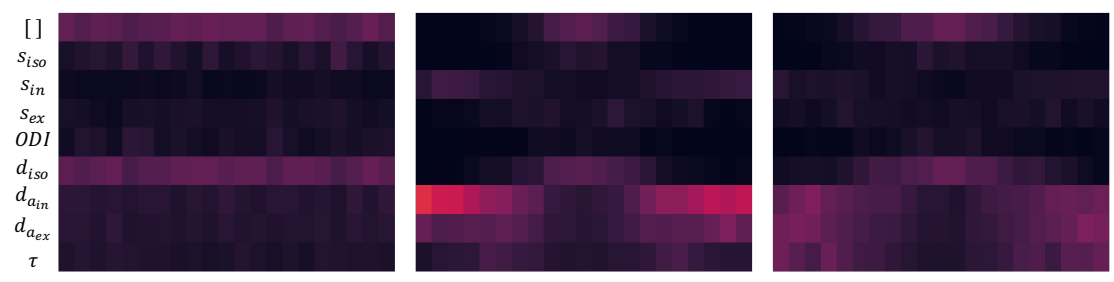

$\triangle O D I$

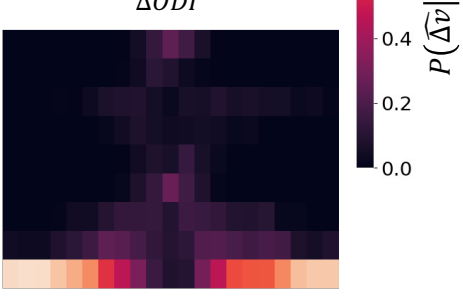

$\Delta \tau$

Figure 10. Sensitivity to the amount of change in the parameters of the standard model. Top: Red curves show the average posterior probability of change in the actual changed parameter as a function of the amount of change. The gray curves show the posterior probability for other parameters. Shaded areas show the 90 percentile range. Larger absolute amount of change results in higher posterior probability for the true parameter change. Change in the signal fraction parameters and $O D I$ is distinguishable for effect sizes as small as 0.05 . However, changes in diffusivity parameters even at very large effect sizes is cluttered with other parameters. Bottom: The same plots shown as heatmaps to better visualize which parameters are confused with each other. 


\section{Estimating the amount of change}

So far we have only examined the posterior probabilities relating to the identity of the parameters that can best explain a change. However our framework also allows us to estimate the posterior probability on the amount of change for each parameter $P(|\Delta v| \mid y, d y, \hat{\Delta v})$ (eq.7). Figure 11 shows the estimated (maximum a posteriori estimation) versus actual change in each parameter for different effect sizes.
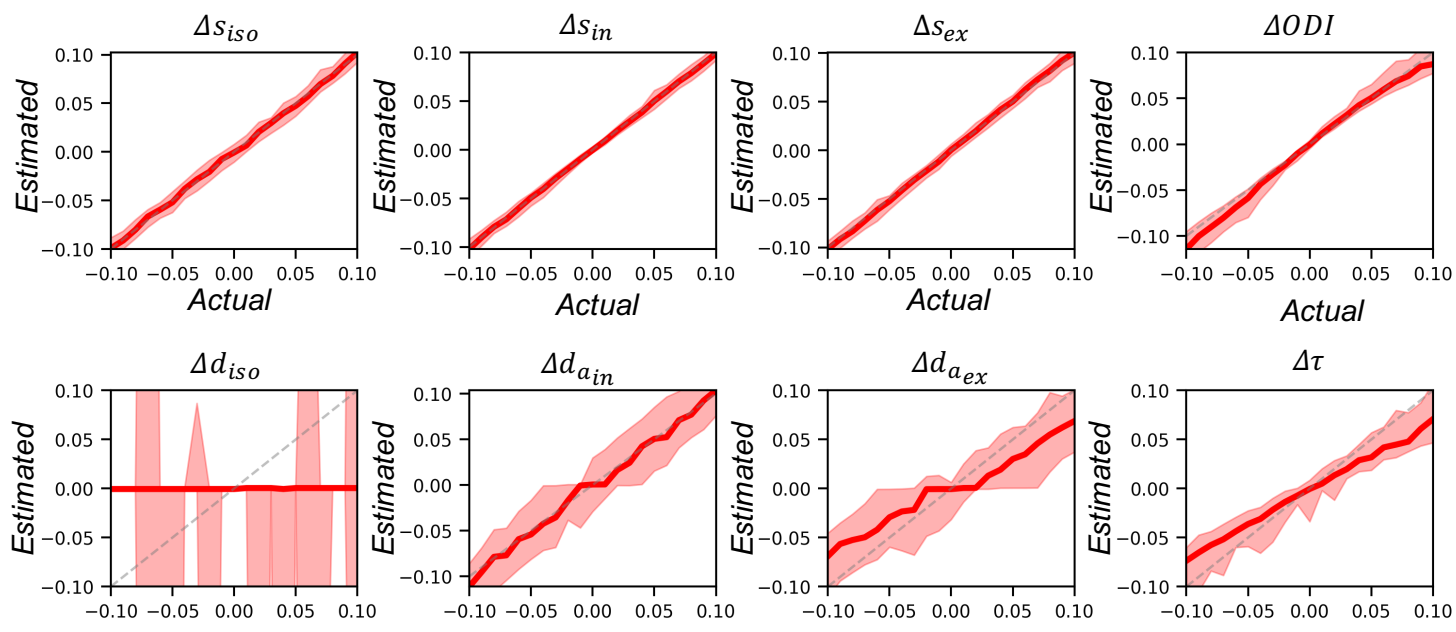

Actual

Actual
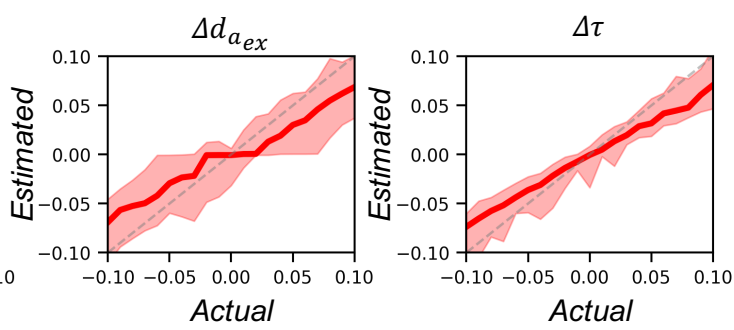

Figure 11. Estimated amount of change in each parameter. Each plot shows the median of the estimated (maximum a posteriori) change vs the actual change in the parameter. The shaded areas show the $10 \%$ interval. The estimated change in the signal fractions follow the identity line (dashed gray line). The estimated change in $d_{i s o}$ is mostly around zero with a high variance as the posterior distribution is very flat and symmetric around zero. The change in $d_{e x, a} \tau$ and $O D I$ is systematically biased at higher effect sizes.

\section{White matter hyperintensities}

Figure 12 shows the observed and estimated amount of change in the summary measurements due to change in each parameter for average data from a small patch in white matter. The last bar in each panel shows the average and standard deviation (across voxels) of the observed amount of change due to WMH normalized by the mean_bO of the baseline measurement. For each parameter the best amount of change given the baseline, observed change and noise covariance is estimated using equation 3 . The bars indicate the amount of change in the measurements that the estimated change in the corresponding parameter can produce. 
The observed change in WMH is an increase in the b0_mean and b1_mean as well as an increase in anisotropy for the b1 shell. This pattern of change is better aligned with a positive change in $s_{e x}$ than in any other parameter.
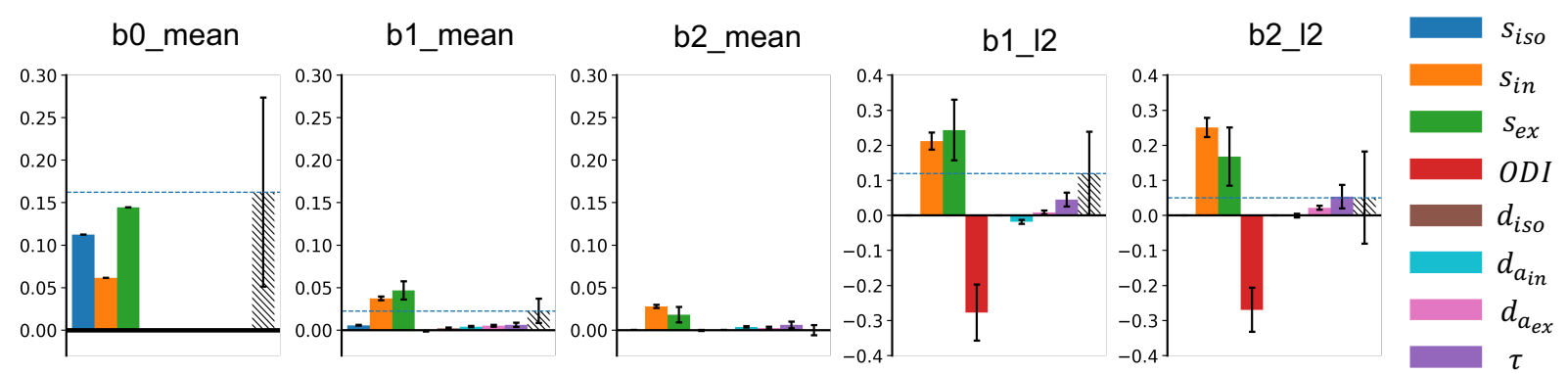

NIII $W M H$

Figure 12. Estimated change vectors along with change in white matter hyperintensities. Each panel shows the estimated amount of change in the measurements if only the corresponding parameter changes, along with the actual observed change in hyperintensities for a patch of voxels in white matter. Each bar is scaled with the best estimated amount of change for that parameter. The observed change in WMH is an increase in the mean-b0 and, to a lesser extent, and increase in mean-bl, and a positive change in the 12 measurements. This is best aligned with the pattern of change that an increase in $s_{e x}$ can produce.

Figure 13 shows the estimated probability of change $P(\hat{\Delta v} \mid \Delta y, y)$ for each parameter of the standard model for an axial slice of the brain in voxels that included more than $10 \mathrm{WMH}$ subjects. These probabilities are normalized to sum up to 1 for each voxel. The colors indicate the probability that a change in the corresponding parameter can explain the observed changes due to WMHs.

Figure 14 shows the best explaining model of change in each voxel in a few axial slices of the brain. In more than $65 \%$ of the voxels, that are mostly in deep white matter, the best model is a change in $s_{e x}$. However, in voxels adjacent to the ventricles, all other models compete and there is not a dominantly winning model. This might be due to a true difference in microstructure in these periventricular voxels, or may be caused by high variability across subjects due to CSF partial volume effects.

Figure 15 shows the estimated amount of change in $s_{e x}$ in voxels where this was the most probable parameter. In most of the voxels an increase in $s_{e x}$ between 0 and 0.4 explains the observed change in WMH. The bottom right panel shows that the amount of change increases with distance from the ventricles, whereas in deep white matter the average amount of change remains 


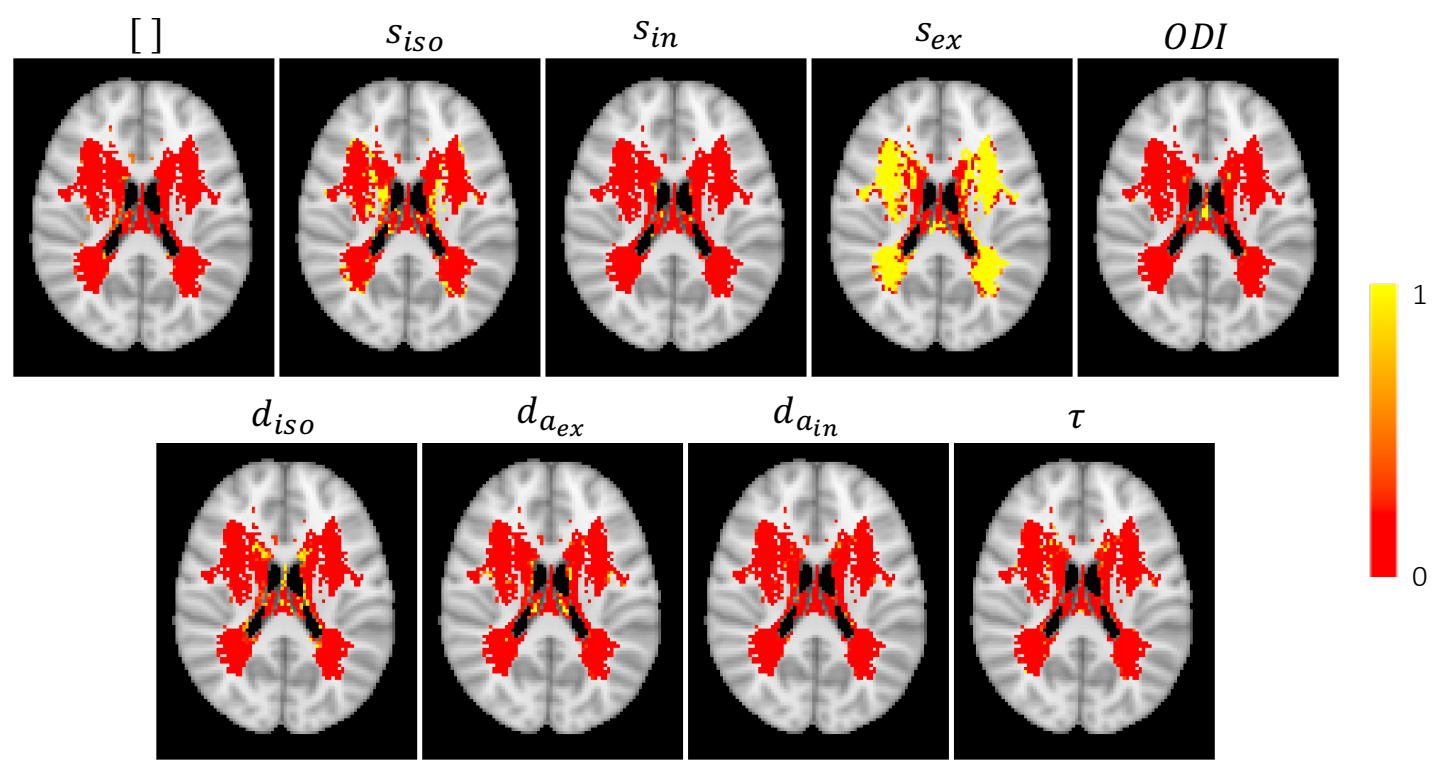

Figure 13. Posterior probability of change in each parameter $P(\hat{\Delta v} \mid \Delta y, y)$. Each map shows the estimated probability that change in the corresponding parameter can explain the observed change in the summary measurements from diffusion MRI between white matter hyperintensities and normally appearing white matter at a single axial slice of the brain. The no change model represents the null model that the change is better explained by noise rather than a change in any one of these parameters. In the majority of the voxels, the change model for $s_{e x}$ has a probability around 1 (yellow) and the remaining parameters are nearly zero(red). This means that a change in $s_{e x}$ is more likely to explain the observed change than any other single parameter change. 

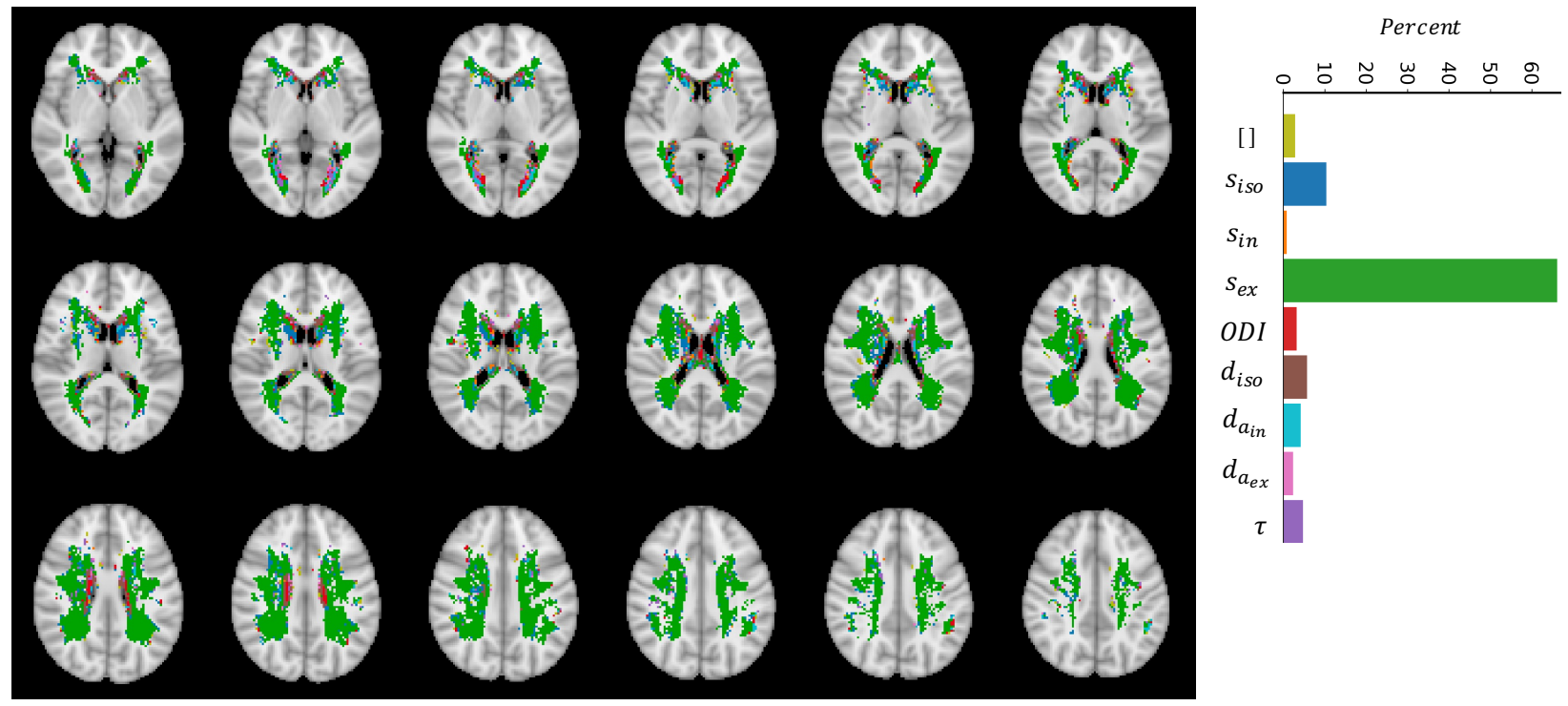

Figure 14. Best explaining model of change in each voxel. The colors indicate which model of change could best explain, i.e. had the highest posterior probability given the observed change in the summary measurements between WMH and normally appearing white matter. In the majority of voxels (68\%) a change in $s_{e x}$ explained the data better than any other model. However, in the regions very close to the ventricles there is no major winning model. This can be either because of high between subject variability or a different type of change that is not captured by the trained models of change.
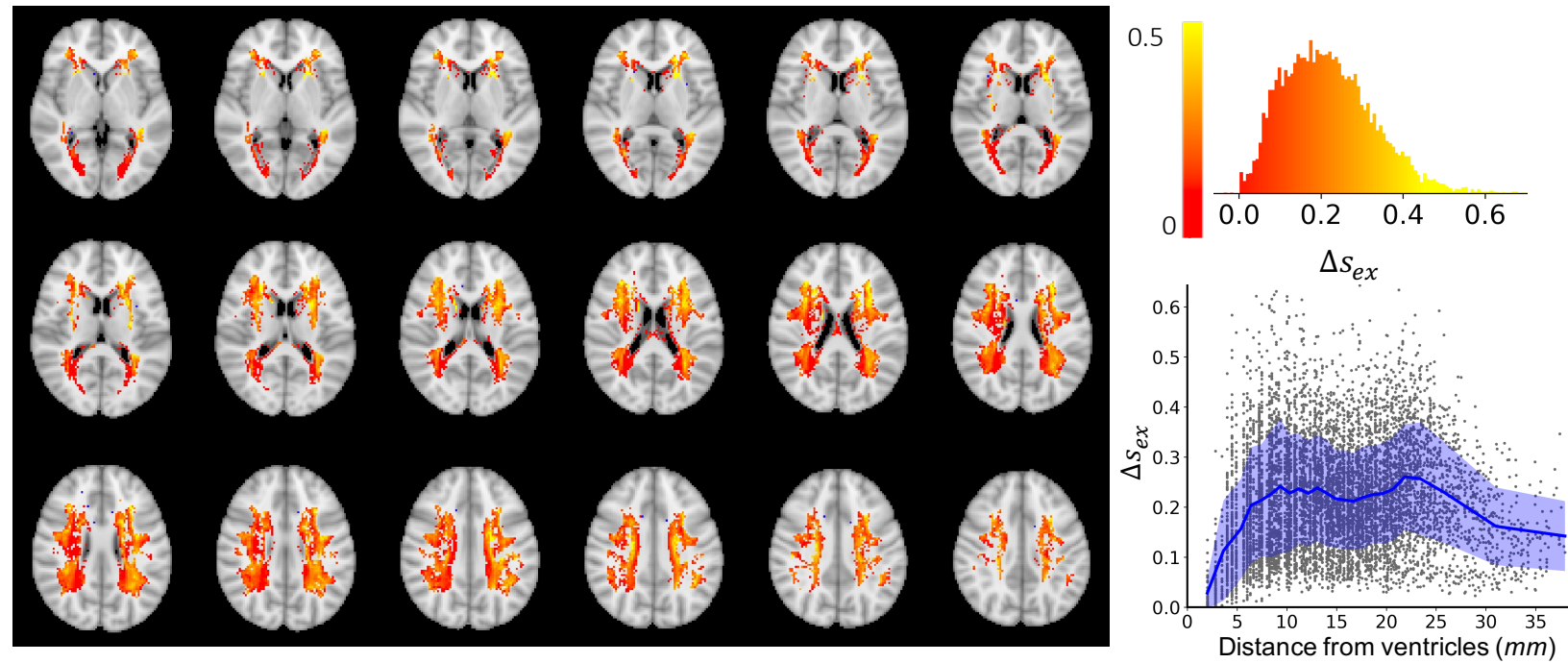

Figure 15. Estimated amount of change in $s_{e x}$. The maps show the estimated amount of change in $s_{e x}$ in voxels where $s_{e x}$ was the best model using a maximum a posteriori estimation $\Delta s_{e x}=\operatorname{argmax} P\left(\Delta v \mid y, \Delta y, \Delta \hat{s}_{e x}\right)$. At most of the voxels the estimated amount of change is positive, meaning that an increase in $s_{e x}$ can explain the change in the summary measurements observed in the WMH voxels. The top right panel shows the distribution of estimated amount of change at the voxels where change in $s_{e x}$ was the best model. Most of the estimated changes are between 0 and 0.4. The bottom right panel shows the amount of change vs the distance (in millimeters) from the ventricles. 


\section{DISCUSSION}

We presented a Bayesian framework to directly infer changes in parameters of a biophysical model from observed changes in a set of measurements. We applied the method to microstructural modelling of diffusion MRI, where biophysical models usually require many free parameters and are often degenerate.

\section{Comparison with model inversion}

The traditional approach to overcome these degeneracies is to constrain some of the parameters to biologically plausible values so that other parameters can be estimated using a conventional measurement (e.g., fixing the diffusivities in NODDI, (Zhang et al. 2012)). Such assumptions reduce the full model parameter space to a restricted subspace, where the model is invertible. This direct inversion approach has the advantage that it gives parameter estimates and that it can model any parameter change in this restricted subspace. However, violation of these assumptions can significantly bias the parameter estimates.

Our proposed approach allows the initial set of parameters to lie anywhere within the full model parameter space (restricted only by broad user-defined priors); and any of these parameters might change. This extra flexibility comes at the price that the parameter changes are assumed to lie along 1D lines in parameter space defined by the user-provided patterns of change $\hat{\Delta v}$. For each of these hypothesized 1D change models, we estimate the posterior probability of such a change as well as the most likely amount given the baseline data and the change in it.

To compare this assumption with that made by direct inversion, let us consider a biophysical model with 8 free parameters. Let us further assume that, due to the limited degrees of freedom in our model, we can only fit 3 out of these 8 parameters. In this case direct inversion would require assuming that the microstructural change is limited to a subset of three parameters, i.e., a 3-dimensional subspace of the full 8-dimensional parameter space. In contrast, BENCH assumes by default that the change is caused by one out of the 8 parameters, which corresponds to the microstructural change lying in one of 8 one-dimensional lines in parameter space. This suggests that if one has prior knowledge of which microstructural parameters are likely to change, it might 
make sense to use direct inversion with those parameters as free parameters. BENCH would have the advantage in a more exploratory approach, where any of the underlying parameters might have changed. However note that this comparison between approaches is complicated by the fact that using model inversion requires setting a subset of the parameters to some fixed value, which might cause a bias in the free parameters if inaccurately fixed (Jelescu et al. 2016; Novikov et al. 2019b).

It is important to note that the user-defined prior distributions for parameters do not directly imply a prior value for the parameters. These priors are used to train the regression models and are required to be wide enough to capture all possible underlying parameter settings. Nevertheless, using broader priors only requires more complex machine learning models that can capture the variation in the relation between the measurements and their derivatives.

In the proposed approach we train the models with simulated data once (without requiring any real data) and use the trained models to estimate the desired probabilities for any real data with the same acquisition protocol. This precomputation saves one from having to integrate over all possible initial parameters when inferring the parameter change in each voxel. Therefore, the inference on real data which only consist of a few $1 \mathrm{~d}$ integrations for each voxel, runs much faster than the non-linear optimizations in alternative inversion approaches.

The results from simulations suggest that we are able to identify changes in signal fraction accurately for the given brain-like measurement. However, there is a considerable confusion in the diffusivities, meaning that the change in these parameters is not distinguishable from one another. These accuracy values depend on the baseline measurements, underlying parameters, and the nature of how each parameter affects the measurements. Nevertheless, an important point is even in the case of full confusion in diffusivities, the results from the proposed approach is more reliable compared to the model inversion with fixed parameters. That is because a wrong prior for the fixed parameters can bias the estimates for other parameters, while in the proposed approach we avoid such assumptions. For example, in NODDI we assume the B0 signal is fixed before and after a change (as a result of the sum constraint on the signal fractions), but in our approach we allow the B0 signal also to change arbitrarily. 
The fact that the approach doesn't require the models to be invertible makes it applicable to studying changes in over-parameterised models or models without closed form analytical solution, e.g. simulation-based models. Such simulation-based models provide the opportunity to explore more complex and realistic models of diffusion in a tissue. There is no limitation in the number of parameters as long as they affect the observed data in some way. If several parameters cause the data to change in the same (or very similar way), this approach will give a list of possible parameters underlying the observed change with a probability associated with each. The resulting probability estimates can be used to eliminate unlikely change scenarios.

We utilized the trained models of change for the parameters of the "standard" model for diffusion to investigate which microstructural changes can explain white matter hyperintensities. The results suggest that the change can be associated with an increase in the extracellular signal. This is in line with other findings using more complex diffusion encodings (Lampinen et al. 2019), who found an increase in the extracellular T2, which would lead to an increase in the extracellular signal contribution. Comparing with the inversion approach, here we did not assume diffusivities are fixed in various brain regions, but we assumed only one of the parameters has changed as a result of white matter hyperintensity. However, it is possible that simultaneous changes in multiple parameters can better explain the change in the data, which could be tested in the same framework with the extended models of change. For example, a model with combination of the parameters might be able to explain a positive change in b0_mean and a negative change in b2_mean as it was observed in some voxels.

\section{Summary measures}

The choice of summary measurements to train change models is arbitrary, but this choice can affect the performance of the model. It is essential that the summary measurements are able to capture enough information from the data such that they are sensitive to changes in the parameters of interest and insensitive to other changes that are not part of the model parameters. For example, in our simulations we did not include the fibre orientation parameters as part of the free parameters, and therefore we required the summary measures to be rotationally invariant. Hence the choice 
of decomposing the signals in each shell into spherical harmonics to extract rotationally invariant summary measurements. Of course one can instead use other signal representations, such as measures derived from the diffusion tensor model, or the kurtosis tensor model, etc, to compute the summary measurements. We chose spherical harmonics over other choices as they are fast to calculate, and the bases are orthogonal which leads to summary measures that capture different aspects of the data.

\section{Future developments}

While in the examples shown here these patterns of change only altered a single parameter at a time, in the current framework the pattern of change can be any vector in parameter space. In the future we plan to extend this framework to allow for parameter changes in 2D or 3D hyperplanes rather than just along 1D lines. However, the dimensionality of these hyperplanes will always be lower than that of the restricted parameter subspace in which parameters can freely change with the direct inversion approach. Note that computing posterior probabilities in a full Bayesian framework allows for comparison between models of change with different complexities without the need for arbitrary regularisation.

In addition, the model of change can be extended to study continuous changes (e.g. ageing), as opposed to discrete group differences as shown in this work. To do so, one first needs to compute the gradient of change in the measurements with respect to the independent variable, e.g. time, using a regression model. Then one can use the chain rule to relate the rate of change in the measurements to the rate of change in the parameters. Such an approach makes modelling continuous change a straightforward extension of this framework.

Although here we mostly show how our method can be applied to detect changes in parameters given the data, our framework can also be used to optimize data acquisition protocols for detecting changes in particular parameters of interest. For example, in the simulations we show that it is difficult to detect a change in the free-diffusion parameter. Our framework can be used to extend the acquisition (e.g. by adding lower bvalues) and, using the output confusion matrices, establish an optimal set of b-shells to enable detection of change in free diffusion. 
Finally, while we applied the framework to the specific problem of studying microstructural changes using diffusion MRI in the brain, the framework is general and can be applied in any field where biophysical models are available. For example, the same approach as described in this paper can be applied to dynamical causal models (DCM) (Friston et al. 2003) for FMRI or MEG/EEG. These are notoriously over-parameterised, but often, are applied in a context where the values of the inferred parameters is of lesser interest than the change in the parameters under different experimental conditions; the ideal scenario for BENCH.

\section{SOFTWARE}

BENCH is an open source software implemented in python and available at https://git. fmrib.ox.ac.uk/hossein/bench.

\section{ACKNOWLEDGEMENTS}

SJ is supported by a Wellcome Senior Fellowship (221933/Z/20/Z), MC and SJ by a Wellcome Collaborative Award (215573/Z/19/Z). The Wellcome Centre for Integrative Neuroimaging is supported by core funding from the Wellcome Trust (203139/Z/16/Z). LG is supported by the National Institute for Health Research (NIHR) Oxford Health Biomedical Research Centre (BRC). UK Biobank Resource under Application 8107 is used in this research. We are grateful to UK Biobank for making the data available, and to all the participants, who made this resource possible by donating their time. The computations were carried out using the Oxford Biomedical Research Computing (BMRC) facilities; a joint development between the Wellcome Centre for Human Genetics and the Big Data Institute that is supported by Health Data Research UK and the NIHR Oxford Biomedical Research Centre. We additionally thank Amy Howard, Paul McCarthy, Mark Woolrich, Karla Miller, Mauro Zucchelli, and Markus Nilsson for their helpful discussions. 


\section{REFERENCES}

Alfaro-Almagro, F., Jenkinson, M., Bangerter, N. K., Andersson, J. L., Griffanti, L., Douaud, G., Sotiropoulos, S. N., Jbabdi, S., Hernandez-Fernandez, M., Vallee, E., et al. (2018). "Image processing and quality control for the first 10,000 brain imaging datasets from uk biobank." Neuroimage, 166, 400-424.

Andersson, J. L., Jenkinson, M., and Smith, S. (2019). "High resolution nonlinear registration with simultaneous modelling of intensities." BioRxiv, 646802.

Assaf, Y., Blumenfeld-Katzir, T., Yovel, Y., and Basser, P. J. (2008). "Axcaliber: a method for measuring axon diameter distribution from diffusion mri." Magnetic Resonance in Medicine: An Official Journal of the International Society for Magnetic Resonance in Medicine, 59(6), $1347-1354$.

Basser, P. J., Mattiello, J., and LeBihan, D. (1994). "Estimation of the effective self-diffusion tensor from the nmr spin echo.” Journal of Magnetic Resonance, Series B, 103(3), 247-254.

Debette, S. and Markus, H. (2010). “The clinical importance of white matter hyperintensities on brain magnetic resonance imaging: systematic review and meta-analysis.” Bmj, 341 .

Friston, K. J., Harrison, L., and Penny, W. (2003). "Dynamic causal modelling." Neuroimage, 19(4), 1273-1302.

Griffanti, L., Zamboni, G., Khan, A., Li, L., Bonifacio, G., Sundaresan, V., Schulz, U. G., Kuker, W., Battaglini, M., Rothwell, P. M., and Jenkinson, M. (2016). "BIANCA (Brain Intensity AbNormality Classification Algorithm): A new tool for automated segmentation of white matter hyperintensities.” NeuroImage, 141, 191-205.

Jelescu, I. O., Veraart, J., Fieremans, E., and Novikov, D. S. (2016). “Degeneracy in model parameter estimation for multi-compartmental diffusion in neuronal tissue." NMR in biomedicine, 29(1), 33.

Jensen, J. H., Helpern, J. A., Ramani, A., Lu, H., and Kaczynski, K. (2005). "Diffusional kurtosis imaging: the quantification of non-gaussian water diffusion by means of magnetic resonance imaging." Magnetic Resonance in Medicine: An Official Journal of the International Society for 
Magnetic Resonance in Medicine, 53(6), 1432-1440.

Kazhdan, M., Funkhouser, T., and Rusinkiewicz, S. (2003). "Rotation invariant spherical harmonic representation of $3 \mathrm{~d}$ shape descriptors." Symposium on geometry processing, Vol. 6, 156-164. Lampinen, B., Szczepankiewicz, F., Novén, M., van Westen, D., Hansson, O., Englund, E., Mårtensson, J., Westin, C.-F., and Nilsson, M. (2019). "Searching for the neurite density with diffusion mri: Challenges for biophysical modeling." Human brain mapping, 40(8), 2529-2545.

Miller, K. L., Alfaro-Almagro, F., Bangerter, N. K., Thomas, D. L., Yacoub, E., Xu, J., Bartsch, A. J., Jbabdi, S., Sotiropoulos, S. N., Andersson, J. L. R., Griffanti, L., Douaud, G., Okell, T. W., Weale, P., Dragonu, I., Garratt, S., Hudson, S., Collins, R., Jenkinson, M., Matthews, P. M., and Smith, S. M. (2016). "Multimodal population brain imaging in the UK Biobank prospective epidemiological study." Nature Neuroscience, 19(11), 1523-1536.

Novikov, D. S., Fieremans, E., Jespersen, S. N., and Kiselev, V. G. (2019a). "Quantifying brain microstructure with diffusion MRI: Theory and parameter estimation." NMR in Biomedicine, 32(4) Publisher: John Wiley \& Sons, Ltd.

Novikov, D. S., Fieremans, E., Jespersen, S. N., and Kiselev, V. G. (2019b). "Quantifying brain microstructure with diffusion mri: Theory and parameter estimation." NMR in Biomedicine, 32(4), e3998.

Novikova, D. S., Veraarta, J., Ileana O. Jelescua, and and Els Fieremans (2018). "Rotationallyinvariant mapping of scalar and orientational metrics of neuronal microstructure with diffusion MRI." NeuroImage, 174, 518-538.

Prins, N. D. and Scheltens, P. (2015). "White matter hyperintensities, cognitive impairment and dementia: an update." Nature Reviews Neurology, 11(3), 157-165.

Reisert, M., Kellner, E., and Kiselev, V. G. (2017). "Disentangling micro from mesostructure by diffusion MRI: A Bayesian approach.” NeuroImage, 147, 964-975 Publisher: Academic Press.

Sotiropoulos, S. N., Behrens, T. E., and Jbabdi, S. (2012). "Ball and rackets: inferring fiber fanning from diffusion-weighted mri." Neuroimage, 60(2), 1412-1425.

Virtanen, P., Gommers, R., Oliphant, T. E., Haberland, M., Reddy, T., Cournapeau, D., Burovski, E., 
Peterson, P., Weckesser, W., Bright, J., van der Walt, S. J., Brett, M., Wilson, J., Millman, K. J., Mayorov, N., Nelson, A. R. J., Jones, E., Kern, R., Larson, E., Carey, C. J., Polat, İ., Feng, Y., Moore, E. W., VanderPlas, J., Laxalde, D., Perktold, J., Cimrman, R., Henriksen, I., Quintero, E. A., Harris, C. R., Archibald, A. M., Ribeiro, A. H., Pedregosa, F., van Mulbregt, P., and SciPy 1.0 Contributors (2020). "SciPy 1.0: Fundamental Algorithms for Scientific Computing in Python." Nature Methods, 17, 261-272.

Wardlaw, J. M., Smith, E. E., Biessels, G. J., Cordonnier, C., Fazekas, F., Frayne, R., Lindley, R. I., T O’Brien, J., Barkhof, F., Benavente, O. R., et al. (2013). "Neuroimaging standards for research into small vessel disease and its contribution to ageing and neurodegeneration." The Lancet Neurology, 12(8), 822-838.

Woolrich, M. W., Jbabdi, S., Patenaude, B., Chappell, M., Makni, S., Behrens, T., Beckmann, C., Jenkinson, M., and Smith, S. M. (2009). "Bayesian analysis of neuroimaging data in fsl." Neuroimage, 45(1), S173-S186.

Zhang, H., Schneider, T., Wheeler-Kingshott, C. A., and Alexander, D. C. (2012). "NODDI: Practical in vivo neurite orientation dispersion and density imaging of the human brain." NeuroImage, 61(4), 1000-1016.

Zhang, Y., Brady, J. M., and Smith, S. (2000). "Hidden markov random field model for segmentation of brain mr image." Medical Imaging 2000: Image Processing, Vol. 3979, International Society for Optics and Photonics, 1126-1137.

Zucchelli, M., Deslauriers-Gauthier, S., and Deriche, R. (2020). “A computational Framework for generating rotation invariant features and its application in diffusion MRI." Medical Image Analysis, 60, 101597. 